\title{
Parameter to State Stability of Control Lyapunov Functions for Hybrid System Models of Robots th
}

\author{
Shishir Kolathaya ${ }^{\mathrm{a}, *}$, Aaron D. Ames ${ }^{\mathrm{a},}$ \\ ${ }^{a}$ Georgia Institute of Technology, 801 Ferst Drive, Atlanta, USA, 30332-0405
}

\begin{abstract}
Model based controllers, by virtue of their dependence on a specific model, are highly sensitive to imperfections in model parameter estimation leading to undesirable behaviors, especially in robots that undergo impacts. With the goal of quantifying the effect of model imperfection on the resulting output behavior from a control Lyapunov function (CLF) based controller, we formally derive a measure for model parameter mismatch and show that a bounded measure leads to an ultimate bound on the CLF. This is also extended to the discrete map by introducing an impact measure. The measure is controller and path dependent, and not just parameter dependent, thereby differentiating it from existing methods. More specifically, if traditional methods yield ultimate boundedness for a bounded parameter uncertainty, the proposed "measure" uses the notion of Input to State Stability (ISS) criterion to establish stability of model based controllers. The main result of this paper establishes that the proposed CLF based controller is parameter to state stable (PSS) for a class of robotic hybrid systems - systems with impulsive effects. These formal results motivate the construction of a robust controller - combining a computed torque term with a traditional PD term - that yields stricter convergence rates and bounds on the errors. This is demonstrated on the bipedal robot AMBER with a modeling error $30 \%$, wherein the stability of the proposed controller is verified in simulation.
\end{abstract}

Keywords: Parameter Uncertainty, Input to State Stability, Hybrid Systems, Control Lyapunov Functions

Preprint submitted to Nonlinear Analysis: Hybrid Systems

September 2, 2016

(C) 2016. This manuscript version is made available under the Elsevier user license http://www.elsevier.com/open-access/userlicense/1.0/ 


\section{Introduction}

There are two main paths for approaching the problem of model parameter uncertainty in mechanical systems: 1. Obtain (usually through exhaustive experimentation) an accurate identification of the model and then adopt a sta-

5 bilizing controller. 2. Develop a robust controller that renders the system stable despite the uncertainty. For the first approach, many methods have been explored in identifying the model parameters involving state estimation, regression, determination and validation in a systematic manner [2, 3]; this often involves substantial and time consuming experimental validation [4. By determining an accurate model, model dependent controllers can be applied to realize accurate tracking and control of such systems. Despite its simplistic nature, the success of this tedious approach typically relies on the accuracy of the estimation while accounting for variations of parameters over time. While these model dependent controllers are able to deliver on the performance (exponential con-

15 vergence, large domains of attraction) promised by the formal controller design process, they are extremely sensitive to changes in the parameters sometimes leading to instability.

There is a significant amount of work in literature that take the second approach, i.e., relax the need for an accurate model [5, 6, 7, 8]. Some of the methods even completely eliminate the requirement of the information of the entire parameter set via adaptive control [9, 10, and via PD and PID regulation and tracking [1, 12, 13, 14, 15, 16, 17]. 18, 19] achieved adaptive control in bipedal robots without considering the impact models. L1 adaptive control was implemented in [20, 21] to yield an ultimate bound on the tracking errors.

25 There is also a significant amount of work done on developing controllers that yield a bounded output error for a bounded parameter uncertainty [5, 6]. While all these methods lead to the development of a robust controller that renders the system stable for a bounded uncertainty, the tracking and regulation performance is sacrificed, which is critical in systems that undergo rapid changes in states - hybrid systems with impulsive effects.

It is important to note that the concept of a bounded error output for a bounded parameter uncertainty has proven to be extremely restrictive on the choice of available controllers. For example, it is a well known fact that the swinging motion of a simple pendulum with zero input (trivial input) is inde35 pendent of the point mass at its end. This simple example demonstrates that

\footnotetext{
A preliminary version of this manuscript was published in the IFAC conference on ADHS 2015 1. The present version shows the Input to State Stability (ISS) property, i.e., zero stability for zero uncertainty, and boundedness for a bounded measure of uncertainty. Rephrasing, the current version shows parameter to state stability of periodic orbits for hybrid robotic systems. The paper also elaborates the proofs of Theorem 1 and 2 in [1] including the detailed procedure for computing the ultimate bounds for the CLF based controller: computed torque $+\mathrm{PD}$.

* Corresponding author

Email address: shishirny@gatech.edu (Shishir Kolathaya)

$U R L$ : www.shishirny.com (Shishir Kolathaya )
} 
we can always realize a space of unbounded model parameter sets that have the exact same response for the given control input. This motivates the need for a formal framework to understand the relationship between model parameter uncertainty and the resulting tracking/regulation performance - especially in the 40 context of hybrid system models of robotic systems. In order to properly quantify this uncertainty that can be formally related to the resulting stability of the system, a measure was defined in [1] and verified in simulation in the robot AMBER. Therefore, the goal of this paper is to expand on [1] and establish and prove stability properties by using the notion of Input to State Stability (ISS) 45 in a formal manner.

For systems of the form $\dot{x}=f(\Theta ; x, u)$, where $\Theta$ represents the parameter set, $x$ represents the state and $u$ the control input, the class of controllers that achieve a desired control objective, e.g., driving $x \rightarrow 0$, can be written via the Control Lyapunov Function (CLF) $V(x)>0$, through the set of control inputs that satisfy the derivative condition that $V$ decreases along solutions:

$$
K=\left\{u \in \mathrm{U}: L_{f} V(x, u) \leq 0\right\} .
$$

Therefore model dependent controllers, like feedback linearization 22 and adaptive control 23 can be reformulated via CLFs which satisfy the condition: $V \rightarrow 0 \Longrightarrow x \rightarrow 0$. Since $\dot{V}(x, u)=\frac{\partial V}{\partial x} f(\Theta ; x, u)$ is a function of the vector field $f$, determination of $u$ depends consequently on the parameters $\Theta$. But, ${ }_{50}$ if the controller (say CLF) that stabilizes the known model is applied on the imperfect model, the resulting dynamics of this imperfect model satisfies the conditions of an Input to State Stable (ISS)-Lyapunov function [24]. The ISSLyapunov function is constructed w.r.t. the input that is a function of the uncertainty. Furthermore, for robotic systems, this function can be written as a linear function of the error in parameters $\Theta$. Therefore, by defining a measure that quantifies the parameter uncertainty as a function of the path and the controller, we can construct robust controllers that yield strict ultimate bounds for the specified uncertainty in the model. Further, as an improvement on the performance, we can construct controllers that use a combination of model based ${ }_{60}$ and non model based controllers (computed torque+PD) to obtain exponential ultimate bounds for hybrid systems.

The primary goal of this paper is to show that, by using the notion of Input to State Stability (ISS), CLFs with a model mismatch can be shown to be Parameter to State Stable for the nonlinear hybrid system model of a robotic

65 system undergoing impacts. In other words, despite the differences in the model, the model based CLF based controller will still yield a bounded output error for a bounded function of parameter uncertainty. To establish this fact, a measure for parameter uncertainty for both the continuous and discrete map will be defined. This will be illustrated through the consideration of a representative robotic system: the bipedal robot AMBER (shown in Fig. 1). There are other approaches like 25] that use control Lyapunov functions to achieve exponential convergence to zero under bounded uncertainty. Our objective is the same, i.e., utilize control Lypapunov functions to obtain exponential convergence, but, to 

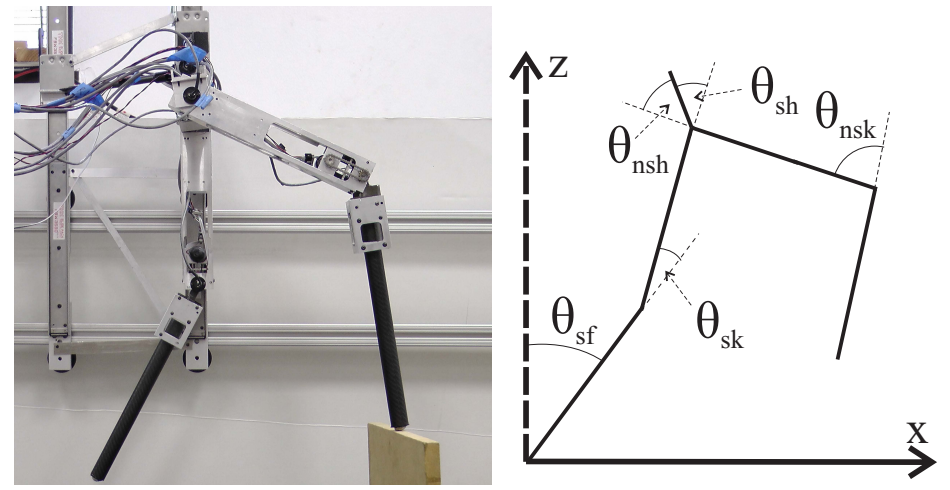

Figure 1: The biped AMBER (left) and the stick figure of AMBER showing the configuration angles (right).

an ultimate bound (in other words, convergence to small acceptable tracking 75 errors). This formal construction helps in obtaining controllers that are not only highly convergent, but also robust to the model mismatch, getting the best from the two worlds. It will be shown with a CLF based controller, computed torque $+\mathrm{PD}$, resulting in a stable walking gait for the robot in simulation.

The paper is structured in the following fashion: Section 2 introduces the

so notion of Input to State Stability (ISS) for both continuous and discrete time systems. The framework to show parameter uncertainty to state stability will be built on this notion. Section 3 introduces the robot model and the control methodology used-Control Lypapunov Functions obtained through the method of computed torque. Section 4 studies this controller in the context of an un85 certain model of the robot and characterizes the resulting uncertain behavior through Lyapunov functions. In Section 5 , the resulting uncertain dynamics exhibited by the robot is measured formally through the construction of a measure that quantifies parameter uncertainty, which is the main formulation of this paper on which the formal results will build. It will be shown that there ${ }_{90}$ is a stronger relationship between the error bounds and the parameter measure than the bounded input bounded output estimate, which motivates the introduction of a robust auxiliary controller: computed torque+PD. This will be utilized for establishing bounds for the entire dynamics, under the assumption of a stable limit cycle in the zero dynamics. This method is extended to hybrid

95 systems through the introduction of an impact measure in Section 6. Under the assumption that the hybrid zero dynamics contains a stable periodic orbit, the computed torque controller appended with the auxiliary input is applied on the model, which results in bounded dynamics of the underactuated hybrid system. The paper concludes with simulation results on a 5-DOF bipedal robot, AMBER, in Section 7, the end result is stable walking even in the presence of parameter uncertainty. 


\section{Preliminaries on Input to State Stability}

In this section we will introduce basic definitions and results related to Input to State Stability (ISS) for a general nonlinear system and then focus on robotic systems in the next section; see [24] for a detailed survey on ISS. A general nonlinear system with outputs is represented in the following fashion:

$$
\begin{aligned}
\dot{x} & =f(x, u), \\
y & =h(x),
\end{aligned}
$$

with $x$ taking values in Euclidean space $\mathbb{R}^{n}$, the input $u \in \mathbb{R}^{k}$, the output $y \in \mathbb{R}^{m}$ for some positive integers $n, m, k$. The mapping $f: \mathbb{R}^{n} \times \mathbb{R}^{k} \rightarrow \mathbb{R}^{n}$ is 105 considered Lipschitz continuous and $f(0,0)=0$ and $h: \mathbb{R}^{n} \rightarrow \mathbb{R}^{m}$ is continuous with $h(0)=0$.

Class $\mathcal{K}_{\infty}$ and $\mathcal{K} \mathcal{L}$ functions. A class $\mathcal{K}_{\infty}$ function is a function $\alpha: \mathbb{R}_{\geq 0} \rightarrow$ $\mathbb{R}_{\geq 0}$ which is continuous, strictly increasing, unbounded, and satisfies $\alpha(0)=0$, and a class $\mathcal{K} \mathcal{L}$ function is a function $\beta: \mathbb{R}_{\geq 0} \times \mathbb{R}_{\geq 0} \rightarrow \mathbb{R}_{\geq 0}$ such that $\beta(r, t) \in$ $\mathcal{K}_{\infty}$ for each $t$ and $\beta(r, t) \rightarrow 0$ as $t \rightarrow \infty$.

Input to State Stability. We can now introduce Input to State Stability to consider the entire dynamics of (2).

Definition 1. The system is Input to State Stable(ISS) if there exists $\beta \in \mathcal{K} \mathcal{L}$, and $\iota \in \mathcal{K}_{\infty}$ such that

$$
\left|x\left(t, x_{0}, u\right)\right| \leq \beta\left(\left|x_{0}\right|, t\right)+\iota\left(\|u\|_{\infty}\right), \quad \forall x_{0}, \forall t \geq 0,
$$

and (2) is considered locally ISS, if the inequality (3) is valid for an open ball of radius $r, x_{0} \in \mathbb{B}_{r}(0)$.

Definition 2. The system is exponential Input to State Stable (e-ISS) if there exists $\beta \in \mathcal{K} \mathcal{L}, \iota \in \mathcal{K}_{\infty}$ and a positive constant $\lambda>0$ such that

$$
\left|x\left(t, x_{0}, u\right)\right| \leq \beta\left(\left|x_{0}\right|, t\right) e^{-\lambda t}+\iota\left(\|u\|_{\infty}\right), \quad \forall x_{0}, \forall t \geq 0,
$$

and (2) is considered locally e-ISS, if the inequality (4) is valid for an open ball of radius $r, x_{0} \in \mathbb{B}_{r}(0)$.

Definition 3. The system is said to hold the asymptotic gain (AG) property if there exists $\iota \in \mathcal{K}_{\infty}$ such that:

$$
\varlimsup_{t \rightarrow \infty}\left|x\left(t, x_{0}, u\right)\right| \leq \iota\left(\|u\|_{\infty}\right), \quad \forall x_{0}, u,
$$

where the $\overline{\lim }_{t \rightarrow \infty}$ denotes the supremum of the limit of $x$ as $t \rightarrow \infty$ (the state $x$ is not required to have a limit). 
Definition 4. The system is said to be zero stable (ZS) for a zero input $u=0$, if there exists $\beta \in \mathcal{K} \mathcal{L}$ such that:

$$
\left|x\left(t, x_{0}, 0\right)\right| \leq \beta\left(\left|x_{0}\right|, t\right), \quad \forall x_{0}, \forall t \geq 0 .
$$
input.

By this definition, ZS $\Longrightarrow$ asymptotic stability of the system with a zero

ISS-Lyapunov functions. We can develop Lyapunov functions that satisfy the ISS conditions and achieve the stability property.

Definition 5. A continuous function $V: \mathbb{R}^{n} \rightarrow \mathbb{R}$ is a storage function if it is positive definite and proper, that is, $V(x) \rightarrow \infty$ as $x \rightarrow \infty$. It is easy to show that $V(x)$ is a storage function if and only if there exist $\underline{\alpha}, \bar{\alpha} \in \mathcal{K}_{\infty}$ such that:

$$
\underline{\alpha}(x) \leq V(x) \leq \bar{\alpha}(x), \quad \forall x \in \mathbb{R}^{n} .
$$

Note that the lower bound amounts to properness and $V(x)>0$ for $x \neq 0$, while the upper bound ensures $V(0)=0$.

Definition 6. An ISS-Lyapunov function for $\dot{x}=f(x, u)$ is a smooth storage function $V: \mathbb{R}^{n} \rightarrow \mathbb{R}_{\geq 0}$ for which there exist functions $\iota, \alpha \in \mathcal{K}_{\infty}$ such that:

$$
\dot{V}(x, u) \leq-\alpha(|x|)+\iota(|u|), \quad \forall x, u .
$$

In other words, an ISS-Lyapunov function is a smooth solution of the inequality of the form (8).

The following lemma establishes the relationship between the ISS-Lyapunov function and the ISS of (2).

Lemma 1. A system is ISS if and only if it admits a smooth ISS-Lyapunov function.

Proof of Lemma 1 is given in [24] and in [26]. In fact the inequality condition can be made stricter by using the exponential estimate:

$$
\dot{V}(x, u) \leq-V(x)+\iota(|u|), \quad \forall x, u .
$$

We can also use the AG property (5) to establish ISS:

Lemma 2. The system is ISS if and only if it is zero stable and AG.

Input to State Stability of Hybrid Systems. We can now introduce ISS and ISS-Lyapunov functions for hybrid systems i.e., specifically systems with impulse effects. A more detailed discussion on ISS notion for hybrid systems can be found in [27, 28, 29, 30. A hybrid system with a single vector field 
(continuous map), and a single discrete map that represents a bipedal robot can be defined as follows (see 31, 32]):

$$
\mathcal{H}=\left\{\begin{aligned}
\dot{x} & =f(x, u), \quad x \in \mathbb{D} \backslash \mathbb{S} \\
x^{+} & =\Delta(x), \quad x \in \mathbb{S},
\end{aligned}\right.
$$

Here $f: \mathbb{R}^{n} \times \mathbb{R}^{k} \rightarrow \mathbb{R}^{n}$ and $\Delta: \mathbb{R}^{n} \rightarrow \mathbb{R}^{n}$ are Lipschitz continuous w.r.t. $x$. Formally, a hybrid system is defined as a tuple: $(\mathbb{D}, \mathcal{U}, \mathbb{S}, \Delta, f)$, where $f, \Delta$ have the usual meaning, $\mathbb{D}, \mathbb{S}$ are the domains of $f, \Delta$ respectively, and $\mathcal{U}$ the set of admissible inputs. This is explained in detail in [30]. For bipedal walking robots, the discrete map shown in 10 is represented by an impact map assuming plastic collisions. The execution of this hybrid system is a tuple $(\Xi, \mathcal{I}, \mathcal{C})$, where $\Xi \subset \mathbb{N}$ is the indexing set, $\mathcal{I}=\{\mathbf{I}\}_{i \in \Xi}$ is the collection of intervals and $\mathcal{C}$ is the set of trajectories. More details can be found in 33 .

For the hybrid system (10), we can obtain $u$ that satisfies the ISS dissipative properties for the continuous map $f$. But, the discrete map does not satisfy this dissipative assumption due to the absence of $u$. On the other hand, due to the periodicity of trajectories in walking, we can realize controllers that render periodic solutions for the hybrid system and the notion of stability of these periodic solutions can be established. Consequently, for the solutions that are periodic with period $T^{*}$, we can define Poincaré maps that motivate the construction of discrete time Lyapunov functions, from which the notion of input to stability

Periodic Orbits and Poincaré maps. The solution $x\left(t, x_{0}\right)$ is periodic if there is a period $T^{*}$ such that $x\left(t+T^{*}, x_{0}\right)=x\left(t, x_{0}\right)$. For this periodic orbit, a Poincaré map $\mathcal{P}: \mathbb{S} \rightarrow \mathbb{S}$ can be defined as: $\mathcal{P}\left(x_{0}\right)=\varphi_{T}\left(\Delta\left(x_{0}\right), u\right)$, where $\varphi$ is the flow of the solution going forward in time, $T$ is the time until the next discrete transition. Therefore, in the neighborhood of the periodic solution, the Poincaré map allows for the reformulation of the hybrid event into a single discrete event. Assume that $\mathcal{O}$ is a periodic orbit in $\mathbb{D}$. If this periodic orbit is locally zero stable, then there is a constant $r>0$ such that if $x$ starts in a ball or radius $r$ defined around $\mathcal{O}, \mathbb{B}_{r}(\mathcal{O})$, then $x(t) \rightarrow \mathcal{O}$ as $t \rightarrow \infty$. If $x^{*} \in \mathcal{O}$ is a point on $\mathbb{S}$ then $\mathcal{P}\left(x^{*}\right)=x^{*}$, which is called the fixed point on the discrete map $\mathcal{P}$. We can construct a discrete time ISS-Lyapunov function that has the following dissipation:

$$
\begin{aligned}
& V\left(\mathcal{P}(x)-x^{*}\right) \leq \lambda V\left(x-x^{*}\right)+\iota(|u|), \quad \forall x \in \mathbb{S} \cap \mathbb{B}_{r}\left(x^{*}\right), u, \\
& \Longrightarrow V\left(\mathcal{P}(x)-x^{*}\right)-V\left(x-x^{*}\right) \leq-(1-\lambda) V\left(x-x^{*}\right)+\iota(|u|),
\end{aligned}
$$

for some $\lambda<1$, and $\iota \in \mathcal{K}_{\infty}$. This motivates using the ISS criterion for the hybrid system 10 as shown in the following Lemma:

Lemma 3. If the Poincaré map $\mathcal{P}$ admits a smooth discrete ISS-Lyapunov function, then the periodic orbit $\mathcal{O}$ of the hybrid system (10) is ISS.

We can make use of this Lemma to show stability of bipedal robotic walking in AMBER (as shown in Fig. 1) under parameter uncertainty. Under the 
assumption that the bipedal robot is zero stable when the model is perfectly known (parameter measure being identically zero), the notion of ISS motivates the introduction of a measure that is a function of the uncertainty in the robot model parameters, and show that the hybrid walking model of the robot is parameter to state stable.

\section{Robot Dynamics and Control}

A robotic system, including a walking robot, can be modeled as $n$-link manipulator. Given the configuration space $\mathbb{Q} \subset \mathbb{R}^{n}$, with the local coordinates $q \in \mathbb{Q}$, and the velocities $\dot{q} \in T_{q} \mathbb{Q}$, the equation of motion of the $n$-DOF robot can be defined as:

$$
D(q) \ddot{q}+C(q, \dot{q}) \dot{q}+G(q)=B \mathrm{~T},
$$

where $D(q) \in \mathbb{R}^{n \times n}$ is the mass inertia matrix of the robot that includes the motor inertia terms, $C(q, \dot{q}) \in \mathbb{R}^{n \times n}$ is the matrix of Coriolis and centrifugal forces, $G(q) \in \mathbb{R}^{n}$ is the gravity vector, $\mathrm{T} \in \mathbb{R}^{k}$ is the torque input and $B \in \mathbb{R}^{n \times k}$ is the mapping from torque to joints. It will be assumed that the mapping $B$ is perfectly known, and is a constant consisting of $1^{\prime} s$ and $0^{\prime} s$.

The system 12 is a nonlinear system where the dynamics can be represented as an affine nonlinear system:

$$
\dot{x}=f_{R}(x)+g_{R}(x) u,
$$

where $x=\left[q^{T}, \dot{q}^{T}\right]^{T}$ and

$$
\begin{gathered}
f_{R}(q, \dot{q})=\left[\begin{array}{c}
1_{n \times n} \\
D^{-1}(q)(-C(q, \dot{q}) \dot{q}-G(q))
\end{array}\right], \\
g_{R}(q, \dot{q})=\left[\begin{array}{c}
0_{n \times k} \\
D^{-1}(q) B
\end{array}\right],
\end{gathered}
$$

$u=\mathrm{T}$ motivated by 2 .

Example 1. As an example, consider the underactuated bipedal robot shown

Fig. 1. The configuration can be defined as: $q=\left(q_{s a}, q_{s k}, q_{s h}, q_{n s h}, q_{n s k}\right)$ corresponding to stance ankle, stance and non-stance knee, stance and nonstance hip angles of the robot. Since the ankle is not actuated, the DOF is $n=5$ and the number of actuators is $k=4$.

Outputs. We will utilize the method of computed torque since it is highly effective for robotic systems [10]. It is also convenient in the context of uncertain models which will be considered in the next section. To realize the controller, outputs are picked which are functions of joint angles referred to as actual outputs $y_{a}: Q \rightarrow \mathbb{R}^{k}$, which are made to track functions termed desired outputs $y_{d}: Q \rightarrow \mathbb{R}^{k}$. The objective is to drive the error $y(q)=y_{a}(q)-y_{d}(q) \rightarrow 0$. These 
outputs are also termed virtual constraints in [34. The outputs are picked such that they are relative degree two outputs (see [35]). Given the output $y$ :

$$
\ddot{y}=\underbrace{\frac{\partial y}{\partial q}}_{J} \ddot{q}+\underbrace{\dot{q}^{T} \frac{\partial^{2} y}{\partial q^{2}}}_{j} \dot{q} .
$$

Since, $k<n$, we add $n-k$ rows to $J$ and $\dot{J}$ to make the co-efficient matrix of $\ddot{q}$ full rank. These rows correspond to the configuration which are underactuated resulting in:

$$
\left[\begin{array}{l}
0 \\
\ddot{y}
\end{array}\right]=\left[\begin{array}{c}
D_{1} \\
J
\end{array}\right] \ddot{q}+\left[\begin{array}{c}
H_{1} \\
\dot{J} \dot{q}
\end{array}\right]
$$

where $H_{1}$ is the first $n-k$ rows of $H(q, \dot{q})=C(q, \dot{q}) \dot{q}+G(q)$, and $D_{1}$ is the first $n-k$ rows of the expression, $D(q)$. These rows correspond to the underactuated degrees of freedom of the robot (corresponding to zero rows of the torque map $B$ ). It should be observed that since the underactuated degrees of freedom have zero torque being applied, the resulting EOM of the robot leads to zero on the left hand side of (15), and hence the choice of rows. Accordingly, we can define the desired acceleration for the robot to be:

$$
\ddot{q}_{d}=\left[\begin{array}{c}
D_{1} \\
J
\end{array}\right]^{-1}\left(\left[\begin{array}{l}
0 \\
\mu
\end{array}\right]-\left[\begin{array}{c}
H_{1} \\
\dot{J} \dot{q}
\end{array}\right]\right)
$$

where $\mu$ is a linear control input. The resulting torque controller that realizes this desired acceleration in the robot can be defined as:

$$
B \mathrm{~T}=D(q) \ddot{q}_{d}+C(q, \dot{q}) \dot{q}+G(q) .
$$

Substituting (17) and (16) in (12) results in linear dynamics: $\ddot{y}=\mu$, where $\mu$ can be chosen to therefore stabilize the $y$ dynamics. We will utilize a CLF based controller to achieve this goal.

Zero Dynamics and Control Lyapunov Function. If we define the vector: $\eta=\left[\begin{array}{c}y \\ \dot{y}\end{array}\right] \in \mathbb{R}^{2 k}$, the output dynamics can be reformulated as:

$$
\dot{\eta}=\underbrace{\left[\begin{array}{cc}
0_{k \times k} & 1_{k \times k} \\
0_{k \times k} & 0_{k \times k}
\end{array}\right]}_{F} \eta+\underbrace{\left[\begin{array}{c}
0_{k \times k} \\
1_{k \times k}
\end{array}\right]}_{G} \mu,
$$

which represent the controllable dynamics of the system. Since, $k<n$ there are states that are not directly controllable which represent the zero dynamics of the system and can be expressed as:

$$
\dot{z}=\Psi(\eta, z),
$$


where $z \in \mathbb{R}^{2(n-k)}$ is the zero dynamic coordinates of the system (see [34).

Consider the candidate Lyapunov function: $V(\eta)=\eta^{T} P \eta$, where $P$ is the solution to the continuous-time algebraic Riccati equation (CARE):

$$
F^{T} P+P F-P G G^{T} P+Q, \quad Q=Q^{T}>0 .
$$

Taking the derivative of $V$ yields:

$$
\dot{V}(\eta)=\eta^{T}\left(F^{T} P+P F\right) \eta+2 \eta^{T} P G \mu .
$$

To find a specific value of $\mu$, we can utilize a minimum norm controller (see [36]) which minimizes $\mu^{T} \mu$ subject to the inequality constraint:

$$
\dot{V}(\eta, \mu)=\eta^{T}\left(F^{T} P+P F\right) \eta+2 \eta^{T} P G \mu \leq-\gamma V(\eta)
$$

185 where $\gamma:=\frac{e^{i g e n} \min (Q)}{\text { eigen }_{\max }(P)}$ is a constant obtained from CARE. Satisfying (21) implies exponential convergence.

We can impose stronger bounds on convergence by constructing a rapidly exponentially stable control lyapunov function (RES-CLF) that can be used to stabilize the output dynamics at a rapid rate through a user defined $\varepsilon>0$ (see 37. for more details).

Definition 7. For the family of continuously differentiable functions, $V_{\varepsilon}$ : $\mathbb{R}^{2 k} \rightarrow \mathbb{R}_{\geq 0}$ is a rapidly exponentially stabilizing control Lyapunov function (RES-CLF) if there exist positive constants $c_{1}, c_{2}, e_{1}>0$ such that for all $0<\varepsilon<1$ and for all $(\eta, z) \in \mathbb{R}^{2 k} \times \mathbb{R}^{2(n-k)}$,

$$
\begin{aligned}
& c_{1}\|\eta\|^{2} \leq V_{\varepsilon}(\eta) \leq \frac{c_{2}}{\varepsilon^{2}}\|\eta\|^{2}, \\
& \inf _{u \in \mathcal{U}}\left[L_{f} V_{\varepsilon}(\eta, z)+L_{g} V_{\varepsilon}(\eta, z) u+\frac{e_{1}}{\varepsilon} V_{\varepsilon}(\eta)\right]<0 .
\end{aligned}
$$

Choosing $P$ and $\varepsilon>0$ :

$$
V_{\varepsilon}(\eta)=\eta^{T}\left[\begin{array}{cc}
\frac{1}{\varepsilon} I & 0 \\
0 & I
\end{array}\right] P\left[\begin{array}{cc}
\frac{1}{\varepsilon} I & 0 \\
0 & I
\end{array}\right] \eta:=\eta^{T} P_{\varepsilon} \eta
$$

It is verified that $V_{\varepsilon}$ is a RES-CLF. By $22, c_{1}, c_{2}>0$ take the minimum and maximum eigenvalues of $P$, respectively. Differentiating 23 yields:

$$
\dot{V}_{\varepsilon}(\eta)=L_{F} V_{\varepsilon}(\eta)+L_{G} V_{\varepsilon}(\eta) \mu,
$$

where $L_{F} V_{\varepsilon}(\eta)=\eta^{T}\left(F^{T} P_{\varepsilon}+P_{\varepsilon} F\right) \eta, L_{G} V_{\varepsilon}(\eta)=2 \eta^{T} P_{\varepsilon} G$.

We can define a minimum norm controller which minimizes $\mu^{T} \mu$ subject to the inequality constraint:

$$
L_{F} V_{\varepsilon}(\eta)+L_{G} V_{\varepsilon}(\eta) \mu \leq-\frac{\gamma}{\varepsilon} V_{\varepsilon}(\eta)
$$

which when satisfied implies exponential convergence. Therefore, we can define a class of controllers $K_{\varepsilon}$ :

$$
K_{\varepsilon}(\eta)=\left\{u \in \mathrm{U}: L_{F} V_{\varepsilon}(\eta)+L_{G} V_{\varepsilon}(\eta) u+\frac{\gamma}{\varepsilon} V_{\varepsilon}(\eta) \leq 0\right\},
$$

which yields the set of control values that satisfies the desired convergence rate. 


\section{Unmodeled Dynamics and State Stability}

195 computed with the given set of parameters will henceforth have over the symbols. Therefore, $D_{a}, C_{a}, G_{a}$ represent the actual model of the robot, and $\hat{D}, \hat{C}$, $\hat{G}$ represent the assumed model of the robot.

It is a well known fact that the inertial parameters of a robot are affine in the EOM (see [38]). Therefore [12) can be restated as:

$$
\mathrm{Y}(q, \dot{q}, \ddot{q}) \Theta=B \mathrm{~T},
$$

where $\mathrm{Y}(q, \dot{q}, \ddot{q})$ is the regressor 38 , and $\Theta$ is the set of base inertial parameters. base inertial parameters. We will consider only the parameters that are difficult to identify, i.e., we will assume that the kinematic parameters, like link lengths, are accurately known. This reduces the set of unknown parameters to only the inertial elements of the robot.

Computed Torque Redefined. The method of computed torque becomes very convenient to apply if the regressor and the inertial parameters are being computed simultaneously. If $\ddot{q}_{d}$ is the desired acceleration vector for the robot, the method of computed torque can be defined as:

$$
B \mathrm{~T}_{c t}=\mathrm{Y}\left(q, \dot{q}, \ddot{q}_{d}\right) \hat{\Theta} .
$$

205

be observed that $(28)$ is just a reformulation of (17). For convenience, the mapping matrix $B$ on the left hand side of $(28)$ will be omitted, i.e., $B \mathrm{~T}_{c t}=\mathrm{T}_{c t}$. Note that it is assumed that the mapping matrix $B$ (including its parameterization) is known to the user. Due to the difference in parameters, it can be observed that the dynamics of the robot becomes different, which is shown be210 low:

Lemma 4. Define:

$$
\Phi=\hat{D}^{-1} \mathrm{Y}(q, \dot{q}, \ddot{q}),
$$

where the dependency of $\Phi$ on $q, \dot{q}, \ddot{q}$ has been suppressed for notational connivance. Similar to (16), if the control law used with the assumed model is:

$$
\ddot{q}_{d}=\left[\begin{array}{c}
\hat{D}_{1} \\
J
\end{array}\right]^{-1}\left(\left[\begin{array}{l}
0 \\
\mu
\end{array}\right]-\left[\begin{array}{c}
\hat{H}_{1} \\
\dot{J} \dot{q}
\end{array}\right]\right),
$$

combined with the computed torque (28), then the resulting dynamics of the robot evolve as:

$$
\ddot{y}=\mu+J \Phi\left(\hat{\Theta}-\Theta_{a}\right) .
$$


Proof. By substituting for $B \mathrm{~T}$ in 12 , we have:

$$
D_{a}(q) \ddot{q}+C_{a}(q, \dot{q}) \dot{q}+G_{a}(q)=\hat{D}(q) \ddot{q}_{d}+\hat{C}(q, \dot{q}) \dot{q}+\hat{G}(q),
$$

by adding and subtracting $\hat{D}(q) \ddot{q}$ on the right hand side of 32 , we have:

$$
\hat{D}(q) \ddot{q}-\hat{D}(q) \ddot{q}_{d}=Y(q, \dot{q}, \ddot{q})\left(\hat{\Theta}-\Theta_{a}\right),
$$

where by substituting for $\ddot{q}_{d}$, the following result is obtained:

$$
\left[\begin{array}{c}
\hat{D}_{1} \ddot{q}+\hat{H}_{1} \\
\ddot{y}
\end{array}\right]=\left[\begin{array}{c}
\hat{D}_{1} \Phi\left(\hat{\Theta}-\Theta_{a}\right) \\
\mu+J \Phi\left(\hat{\Theta}-\Theta_{a}\right)
\end{array}\right] .
$$

The bottom row is the desired result. The top row shows the dynamics of one of the zero coordinates and its relationship with parameter uncertainty.

The dynamics of this uncertain system can be written in the following form:

$$
\begin{aligned}
\dot{\eta} & =F \eta+G \mu+G J \Phi \tilde{\Theta} \\
\dot{z} & =\Psi\left(\Theta_{a} ; \eta, z\right),
\end{aligned}
$$

where $\tilde{\Theta}=\hat{\Theta}-\Theta_{a}$. The dependency of the zero dynamics on the true parameter 215 set $\Theta_{a}$ is explicitly shown. If $\tilde{\Theta}=0$, we could apply 26 to drive $\eta \rightarrow 0$. But since the parameters are uncertain, i.e., $\tilde{\Theta} \neq 0$, the resulting nonlinear dynamics will be observed in the derivative of the Lyapunov function:

$$
\begin{aligned}
\dot{V}_{\varepsilon}(\eta, \mu) & =\dot{\eta}^{T} P_{\varepsilon} \eta+\eta^{T} P_{\varepsilon} \dot{\eta} \\
& =\eta^{T}\left(F^{T} P_{\varepsilon}+P_{\varepsilon} F\right) \eta+2 \eta^{T} P_{\varepsilon} G \mu+2 \eta^{T} P_{\varepsilon} G J \Phi \tilde{\Theta}
\end{aligned}
$$

where $\dot{\eta}$ is obtained via (35). The next section will establish the relationship between parameter uncertainty and the uncertain dynamics appearing in the CLF through the parameter uncertainty measure.

\section{Parameter Uncertainty Measure}

Due to the unmodeled dynamics, applying the controller, $\mu(\eta) \in K_{\varepsilon}(\eta)$, does not result in exponential convergence of the controller. The controller will still yield boundedness based on how the unmodeled dynamics affect $\dot{V}_{\varepsilon}$. The 225 parameter uncertainty measure, $\nu$, that quantifies the ultimate bound on the Lyapunov function $V_{\varepsilon}$ is defined as:

$$
\nu \quad:=\mathrm{Y}(q, \dot{q}, \ddot{q}) \tilde{\Theta} .
$$

It can be observed that: $\mathrm{Y}(q, \dot{q}, \ddot{q}) \tilde{\Theta}=\mathrm{Y}(q, \dot{q}, \ddot{q}) \hat{\Theta}-\mathrm{Y}(q, \dot{q}, \ddot{q}) \Theta_{a}$, which is the difference between the actual and the expected torque being applied on the robot. Therefore, the parameter measure is effectively the difference in torques applied on the robot. It can be observed that the sensitivity and the 
uncertainty analysis w.r.t. the parameters take a similar approach (see [39]), where the partial derivatives of the controller inputs are computed w.r.t. $\Theta$ :

$$
\begin{aligned}
\text { Control Sensitivity } & =\frac{\partial \mathrm{T}}{\partial \Theta}=\mathrm{Y}(q, \dot{q}, \ddot{q}) . \\
\text { Control Uncertainty } & =\frac{\partial \mathrm{T}}{\partial \Theta}\left(\hat{\Theta}-\Theta_{a}\right)=\nu,
\end{aligned}
$$

which justifies the reasoning behind using the nomenclature parameter uncertainty measure. If $\tilde{\Theta}$ is identically a vector of $1^{\prime} s, \nu$ is called parameter sensitivity measure. It must be noted that $\nu$ was called the parameter sensitivity measure in 1] which was changed to the current nomenclature after the review.

Measure to State stability through RES-CLF. By (29) and (37), we have: $\hat{D}^{-1} \nu=\Phi \tilde{\Theta}$. Therefore, (36) can be expressed as:

$$
\dot{V}_{\varepsilon}(\eta, \mu)=\eta^{T}\left(F^{T} P_{\varepsilon}+P_{\varepsilon} F\right) \eta+2 \eta^{T} P_{\varepsilon} G \mu+2 \eta^{T} P_{\varepsilon} G J \hat{D}^{-1} \nu,
$$

which is now a function of $\nu$. This provides an important connection with Lyapunov theory, and the notion of parameter uncertainty is motivated by this observation. In other words, if the path of least $\nu$ is followed, then the convergence of the Lyapunov function to a value very close to zero can be realized. 235 Therefore, we will define the notion of Parameter to State Stability in the following manner:

Definition 8. Assume a ball of radius $r$ around the origin. The system given by (35) is locally parameter to $\eta$ stable, if there exists $\beta \in \mathcal{K} \mathcal{L}$, and $\iota \in \mathcal{K}_{\infty}$ such that:

$$
|\eta(t)| \leq \beta(|\eta(0)|, t)+\iota(\|\nu\|), \quad \forall \eta(0) \in \mathbb{B}_{r}(0), \forall t \geq 0,
$$

and it is locally parameter to state stable (PSS), if

$|(\eta(t), z(t))| \leq \beta(|(\eta(0), z(0))|, t)+\iota(\|\nu\|), \quad \forall(\eta(0), z(0)) \in \mathbb{B}_{r}(0,0), \forall t \geq 0$.

If a suitable controller is applied to [39): $\mu(\eta) \in K_{\varepsilon}(\eta)$, the stability of the Lyapunov function can be achieved as long as the following equation is satisfied:

$$
\dot{V}_{\varepsilon} \leq-\frac{\gamma}{\varepsilon} V_{\varepsilon}+2 \eta^{T} P_{\varepsilon} G J \hat{D}^{-1} \nu \leq 0,
$$

It must be noted that the measure $\nu$ is also a function of the control input $\mu$, resulting in an algebraic loop in 42 . But, by a careful selection of the control input, it is possible to stabilize the dynamics of $\eta$ by the restriction of $\nu$ through the robot model parameters. Therefore, we first assume the bounds on the robot model in the following manner:

$$
\begin{aligned}
& c_{3} \leq\|D\| \leq c_{4},\|C\| \leq c_{5}\|\dot{q}\|,\|G\| \leq c_{6}, \\
& \hat{c}_{3} \leq\|\hat{D}\| \leq \hat{c}_{4},\|\hat{C}\| \leq \hat{c}_{5}\|\dot{q}\|, \quad\|\hat{G}\| \leq \hat{c}_{6} .
\end{aligned}
$$


where $c_{3}-c_{6}, \hat{c}_{3}-\hat{c}_{6}$ are constants (see [40, 41]). Since the outputs are degree one functions of $q$, the Jacobian can also be bounded by the constant: $\|J\| \leq \kappa$. By assuming that $\nu$ is bounded over time, we can establish a parameter to state stable(PSS)-Lyapunov function for the dynamics (39), which is shown in the following Lemma.

Lemma 5. Given a controller $\mu(\eta) \in K_{\varepsilon}(\eta)$, the system 35 is parameter to $\eta$ stable, w.r.t. the parameter input $\nu$.

Proof. Since $V_{\varepsilon}(\eta)$ is bounded by the norms of $\|\eta\|$, we have the following:

$$
\frac{\gamma}{\varepsilon} \eta^{T} P_{\varepsilon} \eta \geq \frac{\gamma}{\varepsilon} c_{1}\|\eta\|^{2}
$$

Considering (42) and the bounds on $P_{\varepsilon}, G, J$ and $\hat{D}$ we have the following inequality:

$$
\dot{V}_{\varepsilon} \leq-\frac{\gamma}{\varepsilon} V_{\varepsilon}+2 \frac{c_{2} \kappa \hat{c}_{3}^{-1}}{\varepsilon^{2}}\|\eta\|\|\nu\| .
$$

The system is 0 stable for $\nu \equiv 0$, and with $\gamma$ replaced with $\gamma_{1}+\gamma_{2}=\gamma$ :

$$
\begin{aligned}
\dot{V}_{\varepsilon} & \leq-\frac{\gamma_{1}+\gamma_{2}}{\varepsilon} V_{\varepsilon}+2 \frac{c_{2} \kappa \hat{c}_{3}^{-1}}{\varepsilon^{2}}\|\eta\|\|\nu\|, \\
\Longrightarrow & \dot{V}_{\varepsilon} \leq-\frac{\gamma_{2}}{\varepsilon} V_{\varepsilon}, \quad \text { for } \frac{\gamma_{1}}{\varepsilon} V_{\varepsilon}(\eta)>2\|\eta\|\|\nu\| \frac{c_{2} \kappa}{\varepsilon^{2} \hat{c}_{3}},
\end{aligned}
$$

which implies that $V$ is decreasing exponentially for $\frac{\gamma_{1}}{\varepsilon} c_{1}\|\eta\|^{2}>2\|\eta\| \frac{c_{2} \kappa}{\varepsilon^{2} \hat{c}_{3}}\|\nu\|$. 245 This satisfies the AG property, and from Lemma 2 it implies parameter to $\eta$ stability w.r.t. $\nu$.

It can be observed that due to $\mathrm{AG}$, the exponential ultimate bound for a nonzero $\|\nu\|_{\infty}$ is given by $\lim _{t \rightarrow \infty}\|\eta(t)\| \leq 2 \frac{c_{2} \kappa}{c_{1} \varepsilon \hat{c}_{3} \gamma_{1}}\|\nu\|_{\infty}$. A good way to reduce the ultimate bound is to increase $\varepsilon$, but, this affects the convergence rate, $\frac{\gamma_{2}}{\varepsilon}$. Consequently Lemma 5 yields a low convergence rate for the output error $\eta$. This affects the stability of hybrid periodic orbits as mentioned in Theorem 2 of [37, which requires $\varepsilon$ to be sufficiently small for stable walking. In order to retain the original convergence rate, $\frac{\gamma}{\varepsilon}$ without sacrificing the ultimate bound, and to nullify the uncertain dynamics separately, we consider an auxiliary input $\bar{\mu}$ satisfying:

$$
B \mathrm{~T}_{c t n}=\hat{D}(q) \ddot{q}_{d}+\hat{C}(q, \dot{q}) \dot{q}+\hat{G}(q)+B \bar{\mu} .
$$

Note that this is not unique and other types of controllers can also be used. Computed torque with linear inputs appended have also been used in [9] in order to realize asymptotic convergence. The resulting dynamics of the outputs then reduces to:

$$
\ddot{y}=\mu+J \hat{D}^{-1} \nu+J \hat{D}^{-1} B \bar{\mu},
$$


which can be obtained by adding $B \bar{\mu}$ in 32 in the proof of Lemma 4 .

By applying (48), 35) will have an extra input $\bar{\mu}$ that yields:

$$
\begin{aligned}
\dot{\eta} & =F \eta+G \mu+G J \hat{D}^{-1} \nu+G J \hat{D}^{-1} B \bar{\mu} \\
\dot{z} & =\Psi\left(\Theta_{a} ; \eta, z\right),
\end{aligned}
$$

Therefore, $\dot{V}_{\varepsilon}$ for the new input can be reformulated as:

$$
\dot{V}_{\varepsilon}(\eta, \mu, \bar{\mu})=\eta^{T}\left(F^{T} P_{\varepsilon}+P_{\varepsilon} F\right) \eta+2 \eta^{T} P_{\varepsilon} G \mu+2 \eta^{T} P_{\varepsilon} G J \hat{D}^{-1}(\nu+B \bar{\mu}) .
$$

Consider the input $\bar{\mu}=-\frac{1}{\bar{\varepsilon}} \Gamma^{T} G^{T} P_{\varepsilon} \eta$, where $\Gamma=J \hat{D}^{-1} B, \bar{\varepsilon}>0$. Then $\mu$ and $\bar{\mu}$ together form the computed torque+PD control on the robot. The end result is a positive semidefinite expression: $\frac{1}{\bar{\varepsilon}} \eta^{T} P_{\varepsilon} G \Gamma \Gamma^{T} G^{T} P_{\varepsilon} \eta \geq 0$, which motives the construction of a positive semidefinite function:

$$
\bar{V}_{\varepsilon}(\eta)=\eta^{T} P_{\varepsilon} G \Gamma \Gamma^{T} G^{T} P_{\varepsilon} \eta=: \eta^{T} \bar{P}_{\varepsilon} \eta .
$$

Using the property of positive semidefiniteness, we can establish new bounds on the outputs. Let $\mathcal{N}\left(\bar{P}_{\varepsilon}\right)$ be the null space of the matrix $\bar{P}_{\varepsilon}$. If $\eta \in \mathcal{N}\left(\bar{P}_{\varepsilon}\right)$, then $\bar{V}_{\varepsilon}(\eta)=0$. Otherwise, for some $c_{7}, c_{8}>0$ :

$$
c_{7}\|\eta\|^{2} \leq \bar{V}_{\varepsilon}(\eta) \leq \frac{c_{8}}{\varepsilon^{4}}\|\eta\|^{2} .
$$

Note that $(52)$ can be used to restrict the uncertain dynamics in (50). Utilizing these constructions, we can define the following class of controllers:

$$
\bar{K}_{\varepsilon, \bar{\varepsilon}}(\eta)=\left\{u \in \mathbb{R}^{k}: 2 \eta^{T} P_{\varepsilon} G J \hat{D}^{-1} B u+\frac{1}{\bar{\varepsilon}} \bar{V}_{\varepsilon}(\eta) \leq 0\right\} .
$$

Lemma 5 can now be redefined to obtain the new exponential ultimate bound for the new control input 47.

Lemma 6. Given the controllers $\mu(\eta) \in K_{\varepsilon}(\eta), \bar{\mu}(\eta) \in \bar{K}_{\varepsilon, \bar{\varepsilon}}(\eta)$, the system (49) is exponential parameter to $\eta$ stable w.r.t. the parameter input $\nu$.

Proof. If $\eta$ is in the null space of the semi definite matrix, as given in (51): $\eta \in \mathcal{N}\left(\bar{P}_{\varepsilon}\right)$, then $\eta^{T} P_{\varepsilon} G=0$ and the uncertainty does not affect $V_{\varepsilon}$ giving the desired result. If $\eta$ does not belong to the null space, then the result can be proved by considering the following constraint:

$$
\frac{1}{\bar{\varepsilon}} \bar{V}_{\varepsilon}(\eta)>2 \frac{c_{2} \kappa \hat{c}_{3}^{-1}}{\varepsilon^{2}}\|\eta\|\|\nu\|,
$$

which gives the AG property: $\lim _{t \rightarrow \infty}\|\eta\| \leq 2 \frac{c_{2} \kappa}{c_{7} \varepsilon^{2} \hat{c}_{3}} \bar{\varepsilon}\|\nu\|_{\infty}=: d_{\eta}\|\nu\|_{\infty}$ implying parameter to $\eta$ stability.

It can be inferred that:

$$
\begin{aligned}
& V_{\varepsilon}(\eta(t)) \leq e^{-\frac{\gamma}{\varepsilon} t} V_{\varepsilon}(\eta(0)) \quad \forall \quad\|\eta(t)\|>d_{\eta}\|\nu\|_{\infty}, \\
& \Rightarrow \quad\|\eta(t)\| \leq \frac{1}{\varepsilon} \sqrt{\frac{c_{2}}{c_{1}}} e^{-\frac{\gamma}{2 \varepsilon} t}\|\eta(0)\| \quad \forall \quad\|\eta(t)\|>d_{\eta}\|\nu\|_{\infty} .
\end{aligned}
$$



$\nu$. Therefore, the gain $\bar{\varepsilon}$ and also the measure norm need to be reasonably small to get sufficient ultimate bounds. This is illustrated very well in Fig. 4 where an almost constant measure (excluding the impacts), and a decreasing $\bar{\varepsilon}$ results in lowering of $V_{\varepsilon}$.

Given the locally Lipschitz continuous feedback law $\mu(\eta)=K_{\varepsilon}(\eta), \bar{\mu}(\eta)=$ $K_{\varepsilon, \bar{\varepsilon}}(\eta)$, we have the following equation:

$$
\begin{aligned}
\dot{\eta} & =F \eta+G \mu(\eta)+G J \hat{D}^{-1} B \bar{\mu}(\eta)+G J \hat{D}^{-1} \nu \\
\dot{z} & =\Psi\left(\Theta_{a} ; \eta, z\right),
\end{aligned}
$$

We can now consider utilizing the ISS notion in underactuated systems given that the zero dynamics of the robot has a locally exponentially stable periodic orbit for the assumed model.

Zero Dynamics. Let $X \subset \mathbb{R}^{2 k}, Z \subset \mathbb{R}^{2(n-k)}$. Let $\varphi_{t}(\eta, z)$ be the flow of (56) with the initial condition $(\eta, z) \in X \times Z$. The flow $\varphi_{t}$ is periodic with period $T^{*}>0$ and a fixed point $\left(\eta^{*}, z^{*}\right)$ if $\varphi_{T^{*}}\left(\eta^{*}, z^{*}\right)=\left(\eta^{*}, z^{*}\right)$. Associated with the periodic flow is the periodic orbit

$$
\mathcal{O}=\left\{\varphi_{t}\left(\eta^{*}, z^{*}\right) \in X \times Z: 0 \leq t \leq T^{*}\right\} .
$$

265 Similarly, we denote the flow of the zero dynamics given by 56 by $\varphi_{t}^{z}$ and for a periodic flow we denote the corresponding periodic orbit by $\mathcal{O}_{Z} \subset Z$. Due to the invariance of the zero dynamics, we have the mapping $\mathcal{O}=\iota_{0}\left(\mathcal{O}_{Z}\right)$, where $\iota_{0}: Z \rightarrow X \times Z$ is the canonical embedding.

Without loss of generality, we can use the norm on $X \times Z$ as the sum of the norms constructed on $X$ and $Z$ separately: $\|(\eta, z)\|=\|\eta\|+\|z\|$. The distance between $(\eta, z)$ and the periodic orbit $\mathcal{O}$ satisfies:

$$
\begin{aligned}
\|(\eta, z)\|_{\mathcal{O}} & =\inf _{\left(\eta^{\prime}, z^{\prime}\right) \in \mathcal{O}}\left\|(\eta, z)-\left(\eta^{\prime}, z^{\prime}\right)\right\| \\
& =\inf _{z^{\prime} \in \mathcal{O}_{Z}}\left\|z-z^{\prime}\right\|+\|\eta-0\| \\
& =\|z\|_{\mathcal{O}_{Z}}+\|\eta\| .
\end{aligned}
$$

The periodic orbit $\mathcal{O}$ is exponentially stable if there are constants $r, \delta_{1}, \delta_{2}>0$ such that if $(\eta, z) \in \mathbb{B}_{r}(\mathcal{O})=\left\{(\eta, z) \in X \times Z:\|(\eta, z)\|_{\mathcal{O}}<r\right\}$ it follows that $\left\|\varphi_{t}(\eta, z)\right\|_{\mathcal{O}} \leq \delta_{1} e^{-\delta_{2} t}\|(\eta, z)\|_{\mathcal{O}}$. Exponential stability of $\mathcal{O}_{Z}$ can also be similarly defined.

We can introduce a theorem that establishes the parameter to state stability of the periodic orbit $\mathcal{O}$ with the assumption that $\mathcal{O}_{Z}$ is exponentially stable.

275 Theorem 1. Assume that the periodic orbit $\mathcal{O}_{z} \subset Z$ is exponentially stable for the assumed model $\hat{\Theta}$. Given the controllers $\mu(\eta) \in K_{\varepsilon}(\eta), \bar{\mu}(\eta) \in \bar{K}_{\varepsilon, \bar{\varepsilon}}(\eta)$ applied on (56), that render exponential $\eta$ stability, then the periodic orbit $\mathcal{O}$ obtained from the canonical embedding is exponential parameter to state stable. 
Proof. Due to the inherent stability of $\eta, 0$ stability of the entire dynamics is directly implied. AG property is established by computing the ultimate bound on the state dynamics $(\eta, z)$. Since $\mathcal{O}_{Z}$ is exponentially stable, there is a Lyapunov function $V_{z}: Z \rightarrow \mathbb{R}_{\geq 0}$ such that in a neighborhood $\mathbb{B}_{r}\left(\mathcal{O}_{Z}\right)$ of $\mathcal{O}_{Z}$ (see [42]) it is exponentially stable. Specific bounds is given by the following:

$$
\begin{aligned}
c_{10}\|z\|_{\mathcal{O}_{Z}}^{2} & \leq V_{z}(z) \leq c_{11}\|z\|_{\mathcal{O}_{Z}}^{2}, \\
\frac{\partial V_{z}}{\partial z} \Psi(\hat{\Theta} ; 0, z) & \leq-c_{12}\|z\|_{\mathcal{O}_{Z}}^{2}, \\
\left\|\frac{\partial V_{z}}{\partial z}\right\| & \leq c_{13}\|z\|_{\mathcal{O}_{Z}},
\end{aligned}
$$

where $c_{10}, c_{11}, c_{12}, c_{13}$ are constants. The zero dynamics of the actual model deviates from the nominal model; we have the following inequality that is inferred from the first row of (34):

$$
\begin{aligned}
\frac{\partial V_{z}}{\partial z} \Psi\left(\Theta_{a} ; 0, z\right) & \leq-c_{12}\|z\|_{\mathcal{O}_{z}}^{2}+\frac{\partial V_{z}}{\partial z}\left(\Psi\left(\Theta_{a} ; 0, z\right)-\Psi(\hat{\Theta} ; 0, z)\right) \\
& \leq-\frac{c_{12}}{2}\|z\|_{\mathcal{O}_{Z}}^{2}-\frac{c_{12}}{2}\|z\|_{\mathcal{O}_{Z}}^{2}+c_{13}\|z\| L_{z}\|\nu\|,
\end{aligned}
$$

for which the exponential upper bound for $z$ can be obtained: $d_{z}\|\nu\|_{\infty}:=$ $\frac{2 c_{13} L_{z}}{c_{12}}\|\nu\|_{\infty} . L_{z}$ is the Lipschitz constant.

${ }^{{ }^{1}}{ }^{2}$ The combined Lyapunov function considering the entire dynamics is given as:

$$
V_{c}(\eta, z)=\sigma V_{z}(z)+V_{\varepsilon}(\eta) .
$$

Upper bounds and lower bounds on $V_{c}$ can be defined as:

$$
\begin{aligned}
& V_{c}(\eta, z) \leq \max \left\{\sigma c_{11}, \frac{c_{2}}{\varepsilon^{2}}\right\}\left(\|z\|_{\mathcal{O}_{Z}}^{2}+\|\eta\|^{2}\right), \\
& V_{c}(\eta, z) \geq \min \left\{\sigma c_{10}, c_{1}\right\}\left(\|z\|_{\mathcal{O}_{Z}}^{2}+\|\eta\|^{2}\right),
\end{aligned}
$$

Therefore, taking the derivative:

$$
\begin{aligned}
\dot{V}_{c}(\eta, z)= & \sigma \frac{\partial V_{z}}{\partial z} \Psi(\hat{\Theta} ; 0, z)+\sigma \frac{\partial V_{z}}{\partial z}\left(\Psi\left(\Theta_{a} ; \eta, z\right)-\Psi\left(\Theta_{a} ; 0, z\right)\right. \\
& \left.+\Psi\left(\Theta_{a} ; 0, z\right)-\Psi(\hat{\Theta} ; 0, z)\right)+\dot{V}_{\varepsilon}(\eta), \\
\leq & -\sigma c_{12}\|z\|_{\mathcal{O}_{Z}}^{2}+\sigma c_{13}\|z\|_{\mathcal{O}_{Z}}\left(L_{q}\|\eta\|+L_{z}\|\nu\|\right)+\dot{V}_{\varepsilon}(\eta),
\end{aligned}
$$

where $L_{q}, L_{z}$ are the Lipschitz constants for $\Psi$ in 35 w.r.t. $\eta$ and $\nu$ respectively. Using (36), 62 can be modified such that sufficient conditions for boundedness can be realized. Using (54) we have the following expression for the Lyapunov function:

$$
\dot{V}_{c} \leq-\sigma \frac{c_{12}}{2}\|z\|_{\mathcal{O}_{Z}}^{2}+\sigma c_{13} L_{q}\|z\|_{\mathcal{O}_{Z}}\|\eta\|-\frac{\gamma}{\varepsilon} V_{\varepsilon}, \quad \forall\|(\eta, z)\|>\left(d_{\eta}+d_{z}\right)\|\nu\|_{\infty} .
$$

To ensure negative definiteness of the $\dot{V}_{c}$ in $63, \sigma$ is picked such that: $\frac{c_{12}}{2} c_{1} \frac{\gamma}{\varepsilon}-$ $\sigma \frac{c_{13}^{2} L_{q}^{2}}{4}>0$, giving the desired result. 


\section{Hybrid Dynamics}

We now extend Theorem 1 to hybrid robotic systems which involve alternating phases of continuous and discrete dynamics. A hybrid system with a single continuous and a discrete event is defined as follows:

$$
\mathscr{H}=\left\{\begin{array}{ccccc}
\dot{\eta} & = & F \eta+G \mu(\eta)+G J \hat{D}^{-1} B \bar{\mu}(\eta)+G J \hat{D}^{-1} \nu, & & \\
\dot{z} & = & \Psi(\Theta ; \eta, z), & \text { if } & (\eta, z) \in \mathbb{D} \backslash \mathbb{S} \\
\eta^{+}= & \Delta_{\eta}\left(\Theta, \eta^{-}, z^{-}\right), & & \\
z^{+}= & \Delta_{z}\left(\Theta, \eta^{-}, z^{-}\right), & & \text {if } & \left(\eta^{-}, z^{-}\right) \in \mathbb{S}
\end{array}\right.
$$

It must be noted that the parameter vector $\Theta$ can be either $\Theta_{a}$ or $\hat{\Theta}$. Since the parameters $\Theta_{a}$ are not known, the output trajectory design is made for the assumed model $\hat{\Theta}$.

It is assumed that $\Psi$ is Lipschitz in both $\nu, \eta$. $\mathbb{D}, \mathbb{S}$ are the domain and switching surfaces and are given by:

$$
\begin{aligned}
& \mathbb{D}=\{(\eta, z) \in X \times Z: h(\eta, z) \geq 0\}, \\
& \mathbb{S}=\{(\eta, z) \in X \times Z: h(\eta, z)=0 \text { and } \dot{h}(\eta, z)<0\},
\end{aligned}
$$

for some continuously differentiable function $h: X \times Z \rightarrow \mathbb{R} . \Delta\left(\Theta, \eta^{-}, z^{-}\right)=$ $\left(\Delta_{\eta}\left(\Theta, \eta^{-}, z^{-}\right), \Delta_{z}\left(\Theta, \eta^{-}, z^{-}\right)\right)$is the reset map representing the discrete dynamics of the system. For the bipedal robot, AMBER, $h$ represents the nonstance foot height and $\Delta$ represents the impact dynamics of the system. Plastic impacts are assumed. For $\left(q^{-}, \dot{q}^{-}\right) \in \mathbb{S}$, being the pre-impact angles and velocities of the robot, the post impact velocity for the assumed model $\dot{\hat{q}}^{+}$, and for the actual model $\dot{q}^{+}$will be obtained from:

$$
\left[\begin{array}{cc}
\hat{D} & -\mathcal{J}^{T} \\
\mathcal{J} & 0
\end{array}\right]\left[\begin{array}{c}
\dot{\hat{q}}^{+} \\
\delta \hat{F}_{\text {imp }}
\end{array}\right]=\left[\begin{array}{c}
\hat{D} \dot{q}^{-} \\
0
\end{array}\right],\left[\begin{array}{cc}
D_{a} & -\mathcal{J}^{T} \\
\mathcal{J} & 0
\end{array}\right]\left[\begin{array}{c}
\dot{q}^{+} \\
\delta F_{i m p}
\end{array}\right]=\left[\begin{array}{c}
D_{a} \dot{q}^{-} \\
0
\end{array}\right]
$$

where $\delta \hat{F}_{i m p}, \delta F_{i m p}$ are the impulsive forces acting from the ground, $\mathcal{J}$ is the Jacobian of the foot where the impulse forces are acting on the robot. By using the Schur complement to get the block matrix inversion we can obtain the post impact velocities as (see [43]):

$$
\begin{aligned}
\dot{\hat{q}}^{+} & =\left(I-\hat{D}^{-1} \mathcal{J}^{T}\left(\mathcal{J} \hat{D}^{-1} \mathcal{J}^{T}\right)^{-1} \mathcal{J}\right) \dot{q}^{-}, \\
\dot{q}^{+} & =\left(I-D_{a}^{-1} \mathcal{J}^{T}\left(\mathcal{J} D_{a}^{-1} \mathcal{J}^{T}\right)^{-1} \mathcal{J}\right) \dot{q}^{-}, \\
\dot{q}^{+}-\dot{\hat{q}}^{+} & =\tilde{D}(q) \dot{q}^{-},
\end{aligned}
$$

where $\tilde{D}(q) \in \mathbb{R}^{n \times n}$ is a long expression obtained after computing the difference 290 between the two post impact velocities. If $D_{a}=\hat{D}, \tilde{D}(q)=0$, which can be used as a candidate for measuring uncertainty during impacts.

Impact Measure. Using the impact model, measuring uncertainty of postimpact dynamics can be achieved by introducing an impact measure, $\nu_{s}$, for 
hybrid systems. It is defined as:

$$
\nu_{s}:=\tilde{D}(q) \dot{q}^{-} .
$$

It should be noted that the impact equations are Lipschitz continuous w.r.t. the impact measure $\nu_{s}$. Accordingly, we have the following bounds on the impact map:

$$
\begin{aligned}
\| \Delta_{\eta}\left(\Theta_{a},\right. & \left.\eta^{-}, z^{-}\right)-\Delta_{\eta}\left(\hat{\Theta}, 0, z^{-}\right) \| \\
& \leq\left\|\Delta_{\eta}\left(\Theta_{a}, \eta^{-}, z^{-}\right)-\Delta_{\eta}\left(\hat{\Theta}, \eta^{-}, z^{-}\right)+\Delta_{\eta}\left(\hat{\Theta}, \eta^{-}, z^{-}\right)-\Delta_{\eta}\left(\hat{\Theta}, 0, z^{-}\right)\right\| \\
& \leq L_{1}\left\|\nu_{s}\right\|+L_{2}\left\|\eta^{-}\right\|,
\end{aligned}
$$

where $L_{1}, L_{2}$ are Lipschitz constants for $\Delta_{\eta}$. Similarly:

$$
\left\|\Delta_{z}\left(\Theta_{a}, \eta^{-}, z^{-}\right)-\Delta_{z}\left(\hat{\Theta}, 0, z^{-}\right)\right\| \leq L_{3}\left\|\nu_{s}\right\|+L_{4}\left\|\eta^{-}\right\|,
$$

where $L_{3}, L_{4}$ are Lipschitz constants for $\Delta_{z}$. In order to obtain bounds on the output dynamics for hybrid periodic orbits, it is assumed that $\mathscr{H}$ has a hybrid zero dynamics for the assumed model, $\hat{\Theta}$, of the robot. More specifically, we assume that $\Delta_{\eta}\left(\hat{\Theta}, 0, z^{-}\right)=0$, so that the surface $Z$ is invariant under the discrete dynamics. The hybrid zero dynamics can be described as:

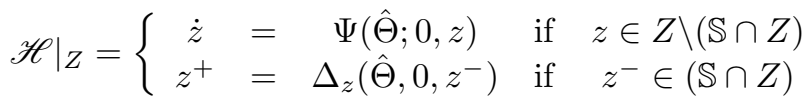

Realizing hybrid zero dynamics (desired trajectories in particular) is in itself a difficult problem and requires large scale nonlinear programming toolboxes which are explained in detail in [44.

Given the hybrid system (64), denote the hybrid flow as $\varphi_{t}\left(\Theta ; \Delta\left(\Theta, \eta^{-}, z^{-}\right)\right)$ with the initial condition $\left(\eta^{-}, z^{-}\right) \in \mathbb{S} \cap Z$. By considering the model estimate $\Theta$, we can define the hybrid flow of (71) as $\varphi_{t}^{z}\left(\hat{\Theta} ; \Delta_{z}\left(\hat{\Theta}, 0, z^{-}\right)\right)$with the initial state $\left(0, z^{-}\right) \in \mathbb{S} \cap Z$. If a periodic orbit $\mathcal{O}_{Z}$ exists in (71), then there exists a periodic flow $\varphi_{t}^{z}\left(\hat{\Theta} ; \Delta_{z}\left(\hat{\Theta}, 0, z^{*}\right)\right)$ of period $T^{*}$ for the fixed point $\left(0, z^{*}\right)$. Through the canonical embedding, the corresponding periodic flow of the periodic orbit $\mathcal{O}$ in (64) will be $\varphi_{t}\left(\hat{\Theta} ; \Delta\left(\hat{\Theta}, 0, z^{*}\right)\right)$. Note that existence of periodic orbits for the assumed model $\hat{\Theta}$ does not guarantee existence for the actual model $\Theta_{a}$. Associated with the hybrid periodic orbit is the Poincaré map $\mathcal{P}: \mathbb{S} \rightarrow \mathbb{S}$ given by:

$$
\mathcal{P}(\Theta ; \eta, z)=\varphi_{T(\Theta ; \eta, z)}(\Theta ; \Delta(\Theta, \eta, z)),
$$

where $\Theta$ can be either $\Theta_{a}$ or $\hat{\Theta}$, and $T$ is the time to impact function defined by:

$$
T(\Theta ; \eta, z)=\inf \left\{t \geq 0: \varphi_{t}(\Theta ; \Delta(\Theta, \eta, z)) \in \mathbb{S}\right\} .
$$

The Poincaré map can be divided into $\eta$ component $\mathcal{P}_{\eta}$, and $z$ component $\mathcal{P}_{z}$ respectively. If the Poincaré map is applied $n$ times, then we denote with the 
superscript $n$ as $\mathcal{P}^{n}(\Theta ; \eta, z)$. Similar to the assumptions made in [37, the implicit function theorem implies that $T$ is well defined in a neighborhood of $\left(\hat{\Theta}, \eta^{*}, z^{*}\right)$. Therefore, $T\left(\hat{\Theta} ; \eta^{*}, z^{*}\right)=T^{*}$ and so $\mathcal{P}\left(\hat{\Theta} ; \eta^{*}, z^{*}\right)=\left(\eta^{*}, z^{*}\right)$. Also, since $\varphi_{t}(\Theta ; \Delta(\Theta, \eta, z))$ is Lipschitz continuous, $T$ is also Lipschitz.

A hybrid periodic orbit $\mathcal{O}_{Z}$, of $\mathscr{H}_{Z}$ can be similarly defined, in which case the corresponding Poincaré map $\rho: \mathbb{S} \cap Z \rightarrow \mathbb{S} \cap Z$ is termed the restricted Poincaré map:

$$
\rho(z)=\varphi_{T_{\rho}(z)}^{z}\left(\hat{\Theta} ; \Delta_{z}(\hat{\Theta}, 0, z)\right)
$$

where $\varphi^{z}$ is the flow of $\dot{z}=\Psi(\hat{\Theta} ; 0, z)$ and $T_{\rho}$ is the restricted time to impact function which is given by $T_{\rho}(z)=T(\hat{\Theta} ; 0, z)$. Without loss of generality, we can assume that $\hat{\Theta}=0, \eta^{*}=0, z^{*}=0$. For ease of notations let $\Theta=\Theta_{a}-\hat{\Theta}=\Theta_{a}$ 1. The following Lemma will introduce the relationship between time to impact, 305 Poincaré functions with the state $\eta$ and the impact measure $\nu_{s}$.

Lemma 7. Let $\mathcal{O}_{Z}$ be the periodic orbit of the hybrid zero dynamics $\left.\mathscr{H}\right|_{Z}$ transverse to $\mathbb{S} \cap Z$ for the nominal model $\hat{\Theta}(\Theta=0)$. Given $\Theta_{a}(\Theta \neq 0)$, and given the controllers $\mu(\eta) \in K_{\varepsilon}(\eta), \bar{\mu}(\eta) \in \bar{K}_{\varepsilon, \bar{\varepsilon}}(\eta)$ applied on the hybrid system 64), then for $r>0$ such that $(\eta, z) \in \mathbb{B}_{r}(0,0)$ and $\|\eta\|>d_{\eta}\|\nu\|_{\infty}$, there exist

$$
\begin{aligned}
\left\|T(\Theta ; \eta, z)-T_{\rho}(z)\right\| & \leq A_{1}\|\eta\|+A_{2}\|\nu\|_{\max }, \\
\left\|\mathcal{P}_{z}(\Theta ; \eta, z)-\rho(z)\right\| & \leq A_{3}\|\eta\|+A_{4}\|\nu\|_{\max }, \quad\|\nu\|_{\max }=\max \left\{\|\nu\|_{\infty},\left\|\nu_{s}\right\|\right\} .
\end{aligned}
$$

Proof. 75 is proved by constructing an auxiliary time to impact function $T_{B}$ that is Lipschitz continuous and then relate it to $T$. Let $\mu_{1} \in \mathbb{R}^{2(n-k)}, \mu_{2} \in \mathbb{R}^{2 k}$ be constant vectors and let $\varphi_{t}^{z}\left(\Delta_{z}\left(0,0, z_{0}\right)\right)$ be the solution of $\dot{z}=\Psi(0 ; 0, z)$ with $z(0)=\Delta_{z}\left(0,0, z_{0}\right)$. Define:

$$
T_{B}\left(\mu_{1}, \mu_{2}, z\right)=\inf \left\{t \geq 0: h\left(\mu_{1}, \varphi_{t}^{z}\left(\Delta_{z}(0,0, z)\right)+\mu_{2}\right)=0\right\},
$$

which is nothing but equation (55) of [37] with the inclusion of model parameters. It follows that $T_{B}(0,0, z)=T_{\rho}(z)$. By construction, $T_{B}$ is Lipschitz continuous. We have,

$$
\left\|T_{B}\left(\mu_{1}, \mu_{2}, z\right)-T_{\rho}(z)\right\| \leq L_{B}\left(\left\|\mu_{1}\right\|+\left\|\mu_{2}\right\|\right),
$$

where $L_{B}$ is the local Lipschitz constant. We note that $T(\Theta ; \eta, z)$ is continuous and therefore there exists $r>0$ such that for all $\mathbb{B}_{r}(0,0) \cap \mathbb{S}: c_{15} T^{*} \leq$ $T(\Theta ; \eta, z) \leq c_{16} T^{*}$, where $0<c_{15}<1$ and $c_{16}>1$. Let $\left(\eta_{1}(t), z_{1}(t)\right)$ satisfy $\dot{z}_{1}=\Psi\left(\Theta ; \eta_{1}(t), z_{1}(t)\right)$ with $\eta_{1}(0)=\Delta_{\eta}(\Theta, \eta, z)$ and $z_{1}(0)=\Delta_{z}(\Theta, \eta, z)$. Simi315 larly let $z_{2}(t)$ satisfy $\dot{z}_{2}(t)=\Psi\left(0 ; 0, z_{2}(t)\right)$ such that $z_{2}(0)=\Delta_{z}(0,0, z)$. We can now determine $\mu_{1}, \mu_{2}$.

\footnotetext{
${ }^{1}$ It is safe to assume $\hat{\Theta}=0$ here because we are interested in capturing the uncertainty in the dynamics (difference) and not the actual dynamics by itself.
} 
The bounds on the Lyapunov function can be given as $\left\|\eta_{1}(0)\right\|=\| \Delta_{\eta}(\Theta, \eta, z)-$ $\Delta_{\eta}(0,0, z)\left\|\leq L_{1}\right\| \nu_{s}\left\|+L_{2}\right\| \eta \|$, which is obtained through (69). Since $\left\|\eta_{1}\right\|>d$, by using (55) we have:

$$
\left\|\eta_{1}(t)\right\|_{t=T(\Theta ; \eta, z)} \leq \sqrt{\frac{c_{2}}{c_{1}}} \frac{1}{\varepsilon} e^{-\frac{\gamma}{2 \varepsilon} c_{15} T^{*}}\left(L_{1}\left\|\nu_{s}\right\|+L_{2}\|\eta\|\right),
$$

which yields the value of $\left\|\mu_{1}\right\|$. To obtain $\left\|\mu_{2}\right\|$, we use the Gronwall-Bellman argument (similar to page 8 in [37]). We know that:

$$
z_{1}(t)-z_{2}(t)=z_{1}(0)-z_{2}(0)+\int_{0}^{t} \Psi\left(\Theta ; \eta_{1}(\tau), z_{1}(\tau)\right)-\Psi\left(0 ; 0, z_{2}(\tau)\right) d \tau,
$$

and therefore by using $(70)$ and using the property of Lipschitz continuity of $\Psi$ :

$$
\begin{gathered}
\left\|z_{1}(t)-z_{2}(t)\right\| \leq L_{3}\left\|\nu_{s}\right\|+L_{4}\|\eta\|+\int_{0}^{t} L_{q}\left(\left\|\eta_{1}(\tau)\right\|+\left\|z_{1}(\tau)-z_{2}(\tau)\right\|\right)+L_{z}\|\nu\| d \tau \\
\leq \quad L_{3}\left\|\nu_{s}\right\|+L_{4}\|\eta\|+\frac{2}{\gamma} \sqrt{\frac{c_{2}}{c_{1}}} L_{q}\left(L_{1}\left\|\nu_{s}\right\|+L_{2}\|\eta\|\right)+c_{16} T^{*} L_{z}\|\nu\|_{\infty} \\
\quad+\int_{0}^{t} L_{q}\left(\left\|z_{1}(\tau)-z_{2}(\tau)\right\|\right) d \tau
\end{gathered}
$$

where 777 is integrated and substituted in the above equation. By GronwallBellman inequality,

$$
\begin{gathered}
\left\|z_{1}(t)-z_{2}(t)\right\| \leq\left(C_{2}\|\eta\|+C_{3}\|\nu\|_{\max }\right) e^{L_{q} t} \\
C_{2}=\frac{2}{\gamma} \sqrt{\frac{c_{2}}{c_{1}}} L_{q} L_{2}+L_{4}, \quad C_{3}=2 \max \left\{\frac{2}{\gamma} \sqrt{\frac{c_{2}}{c_{1}}} L_{q} L_{1}+L_{3}, c_{16} T^{*} L_{z}\right\} .
\end{gathered}
$$

325 Therefore, $\left\|\mu_{2}\right\| \leq\left\|z_{1}\left(c_{16} T^{*}\right)-z_{2}\left(c_{16} T^{*}\right)\right\|$ by substituting for the upper bound on $T$. Proof of 75 , can now be obtained by substituting for $\left\|\mu_{1}\right\|,\left\|\mu_{2}\right\|$.

To prove (76), define:

$$
C_{4}=\max _{c_{15} T^{*} \leq t \leq c_{16} T^{*}}\left\|\Psi\left(0 ; 0, z_{2}(t)\right)\right\|
$$

it then follows that:

$$
\begin{aligned}
& \left\|\mathcal{P}_{z}(\Theta ; \eta, z)-\rho(z)\right\| \leq\left\|z_{1}(0)-z_{2}(0)\right\| \\
& \quad+\int_{0}^{T(\Theta ; \eta, z)}\left\|\Psi\left(\Theta ; \eta_{1}(\tau), z_{1}(\tau)\right)-\Psi\left(0 ; 0, z_{2}(\tau)\right)\right\| d \tau \\
& \quad+\int_{T(\Theta ; \eta, z)}^{T_{\rho}(z)}\left\|\Psi\left(0 ; 0, z_{2}(\tau)\right)\right\| d \tau,
\end{aligned}
$$

which results in the following inequality:

$$
\left\|\mathcal{P}_{z}(\Theta ; \eta, z)-\rho(z)\right\| \leq\left\|z_{1}\left(c_{16} T^{*}\right)-z_{2}\left(c_{16} T^{*}\right)\right\|+C_{4}\left\|T(\Theta ; \eta, z)-T_{\rho}(z)\right\| .
$$

Collecting the terms together yields the desired result. 
Main Theorem. We can now introduce the main theorem of the paper. Similar to the continuous dynamics, it is assumed that the periodic orbit $\mathcal{O}_{Z}$ is exponentially stable in the hybrid zero dynamics.

Theorem 2. Let $\mathcal{O}_{Z}$ be an exponentially stable periodic orbit of the hybrid zero 335 dynamics $\left.\mathscr{H}\right|_{Z}$ transverse to $\mathbb{S} \cap Z$ for the nominal model $\hat{\Theta}(\Theta=0)$. Given the actual model $\Theta_{a}(\Theta \neq 0)$ and the controllers $\mu(\eta) \in K_{\varepsilon}(\eta), \bar{\mu}(\eta) \in \bar{K}_{\varepsilon, \bar{\varepsilon}}(\eta)$ for the hybrid system $\mathscr{H}$ given by (64), then for the ball of radius $r>0$ such that $(\eta, z) \in \mathbb{B}_{r}(0,0)$, there exists $\delta>0$ such that for $\|\nu\|<\delta,\left\|\nu_{s}\right\|<\delta$ the periodic orbit $\mathcal{O}$ is exponential parameter to state stable.

${ }_{340}$ Proof. Results of Lemma 7 and the exponential stability of $\mathcal{O}_{Z}$ imply that there exists $r>0$ such that $\rho: \mathbb{B}_{r}(0) \cap(\mathbb{S} \cap Z) \rightarrow \mathbb{B}_{r}(0) \cap(\mathbb{S} \cap Z)$ is well defined for all $z \in \mathbb{B}_{r}(0) \cap(\mathbb{S} \cap Z)$ and $z_{j+1}=\rho\left(z_{j}\right)$ is locally exponentially stable, i.e., $\left\|z_{j}\right\| \leq N \xi^{j}\left\|z_{0}\right\|$ for some $N>0,0<\xi<1$ and all $j \geq 0$. Therefore, by the converse Lyapunov theorem for discrete systems, there exists a Lyapunov function $V_{\rho}$, defined on $\mathbb{B}_{r}(0) \cap(\mathbb{S} \cap Z)$ for some $r>0$ (possibly smaller than the previously defined $r$ ), and positive constants $c_{17}, c_{18}, c_{19}, c_{20}$ such that:

$$
\begin{aligned}
c_{17}\|z\|^{2} \leq V_{\rho}(z) & \leq c_{18}\|z\|^{2}, \\
V_{\rho}(\rho(z))-V_{\rho}(z) & \leq-c_{19}\|z\|^{2}, \\
\left|V_{\rho}(z)-V_{\rho}\left(z^{\prime}\right)\right| & \leq c_{20}\left\|z-z^{\prime}\right\| .\left(\|z\|+\left\|z^{\prime}\right\|\right) .
\end{aligned}
$$

Zero stability (perfect model) is valid by default due to the construction (hybrid zero dynamics and RES-CLF with sufficiently small $\varepsilon$ provide exponential convergence to $\mathcal{O}$, see [37]). To prove $A G$ property it should be first ensured that the region defined by $\varlimsup_{t \rightarrow \infty}\|(\eta(t), z(t))\|$ must be within the bounds defined by $r$. Therefore it is required that the ultimate bound is less than $r$ :

$$
\left(d_{\eta}+d_{z}\right)\|\nu\|_{\infty}<r, \quad \delta_{r}:=\frac{r}{\left(d_{\eta}+d_{z}\right)},
$$

which gives the allowable upper bound $\delta_{r}$ on $\|\nu\|_{\infty}$. It is also required that the post impact states are also in $\mathbb{B}_{r}(0,0)$. Therefore, a second upper bound $\delta_{I}$ is given as follows:

$$
\begin{array}{r}
\|\Delta(\Theta ; \eta, z)\| \leq L_{\Delta_{1}}\left\|\nu_{s}\right\|+L_{\Delta_{2}}\|(\eta, z)\| \leq r, \\
\Longrightarrow \delta_{I}:=\frac{r}{L_{\Delta_{1}}+L_{\Delta_{2}}\left(d_{\eta}+d_{z}\right)},
\end{array}
$$

$L_{\Delta_{1}}, L_{\Delta_{2}}$ are Lipschitz constants.

For the RES-CLF $V_{\varepsilon}$, denote its restriction to the switching surface by

$$
V_{\varepsilon, \eta}(\eta)=\left.V_{\varepsilon}\right|_{\mathbb{S}}(\eta)=V_{\varepsilon}(\eta), \quad \eta \in \mathbb{S} .
$$

With these two Lyapunov functions we define the following candidate Lyapunov function:

$$
V_{P}(\eta, z)=V_{\rho}(z)+\sigma V_{\varepsilon, \eta}(\eta),
$$


defined on $\mathbb{B}_{r}(0,0) \cap \mathbb{S}$. The lower and upper bounds on $V_{P}$ are:

$$
\min \left\{c_{17}, \sigma c_{1}\right\}\|(\eta, z)\|^{2} \leq V_{P}(\eta, z) \leq \max \left\{c_{18}, \sigma \frac{c_{2}}{\varepsilon^{2}}\right\}\|(\eta, z)\|^{2} .
$$

The idea is to show that there exists a bounded region into which the dynamics of the robot exponentially converge. If the outputs enter this region then it stays for all time even through impacts. We know that $\eta_{1}(0)=\Delta_{\eta}(\Theta, \eta, z)$. if $\left\|\eta_{1}(0)\right\|<d_{\eta}\|\nu\|_{\infty}$, then the boundedness is verified. Considering the case when the impact map takes the outputs outside the bounded region, utilizing (55), and picking the lower bound on the time to impact function:

$$
\begin{aligned}
V_{\varepsilon, \eta}\left(\mathcal{P}_{\eta}(\Theta ; \eta, z)\right) & \leq \frac{c_{2}}{\varepsilon^{2}} e^{-\frac{\gamma}{\varepsilon} T(\Theta ; \eta, z)}\left\|\Delta_{\eta}(\Theta, \eta, z)\right\|^{2} \\
& \leq \frac{c_{2}}{\varepsilon^{2}} e^{-\frac{\gamma}{\varepsilon} T(\Theta ; \eta, z)}\left(L_{1}\left\|\nu_{s}\right\|+L_{2}\|\eta\|\right)^{2}
\end{aligned}
$$

Taking $A_{5}=\frac{c_{2}}{\varepsilon^{2}} e^{-\frac{\gamma}{\varepsilon} c_{15} T^{*}}$, we have the following:

$$
\begin{aligned}
V_{\varepsilon, \eta}\left(\mathcal{P}_{\eta}(\eta, z)\right)-V_{\varepsilon, \eta}(\eta) & \leq A_{5}\left(L_{1}\left\|\nu_{s}\right\|+L_{2}\|\eta\|\right)^{2}-c_{1}\|\eta\|^{2} . \\
& \leq A_{5}\left(L_{1}\|\nu\|_{\max }+L_{2}\|\eta\|\right)^{2}-c_{1}\|\eta\|^{2} .
\end{aligned}
$$

Since the origin is an exponentially stable equilibrium for $z_{j+1}=\rho\left(z_{j}\right)$, we have the following inequalities:

$$
\begin{aligned}
\left\|\mathcal{P}_{z}(\Theta ; \eta, z)\right\| & =\left\|\mathcal{P}_{z}(\Theta ; \eta, z)-\rho(z)+\rho(z)-\rho(0)\right\| \\
& \leq A_{3}\|\eta\|+A_{4}\|\nu\|_{\max }+L_{\rho}\|z\| \\
\|\rho(z)\| & \leq N \xi\|z\|,
\end{aligned}
$$

where $L_{\rho}$ is the Lipschitz constant for $\rho$. Therefore:

$$
\begin{aligned}
V_{\rho}\left(\mathcal{P}_{z}(\Theta ; \eta, z)\right)-V_{\rho}(\rho(z)) \leq c_{20}\left(A_{3}\|\eta\|+A_{4}\|\nu\|_{\max }\right) \\
\left(A_{3}\|\eta\|+A_{4}\|\nu\|_{\max }+\left(L_{\rho}+N \xi\right)\|z\|\right) .
\end{aligned}
$$

It follows that:

$$
V_{\rho}\left(\mathcal{P}_{z}(\Theta ; \eta, z)\right)-V_{\rho}(z)=V_{\rho}\left(\mathcal{P}_{z}(\Theta ; \eta, z)\right)-V_{\rho}(\rho(z))+V_{\rho}(\rho(z))-V_{\rho}(z),
$$

and the expressions in (90) and in (81) can be substituted. Combining the entire Lyapunov function we have:

$$
V_{P}(\mathcal{P}(\Theta ; \eta, z))-V_{P}(\eta, z) \leq-\left[\begin{array}{c}
\|\eta\| \\
\|z\| \\
\|\nu\|_{\max }
\end{array}\right]^{T} \Lambda_{\mathscr{H}}\left[\begin{array}{c}
\|\eta\| \\
\|z\| \\
\|\nu\|_{\max }
\end{array}\right]
$$

where the symmetric matrix $\Lambda_{\mathscr{H}} \in \mathbb{R}^{3 \times 3}$, with the upper triangular entries 
being:

$$
\begin{aligned}
a_{1}=\Lambda_{\mathscr{H}}(1,1) & =\sigma\left(c_{1}-A_{5} L_{2}^{2}\right)-c_{20} A_{3}^{2} \\
-a_{2}= & \Lambda_{\mathscr{H}}(1,2)=-\frac{c_{20} A_{3}}{2}\left(L_{\rho}+N \xi\right) \\
-a_{3}=\Lambda_{\mathscr{H}}(1,3) & =-c_{20} A_{3} A_{4}-\sigma A_{5} L_{1} L_{2} \\
a_{4}=\Lambda_{\mathscr{H}}(2,2) & =c_{19} \\
-a_{5}=\Lambda_{\mathscr{H}}(2,3) & =-\frac{c_{20} A_{4}}{2}\left(L_{\rho}+N \xi\right) \\
-a_{6}=\Lambda_{\mathscr{H}}(3,3) & =-\sigma A_{5} L_{1}^{2}-c_{20} A_{4}^{2} .
\end{aligned}
$$

(92) is in the form of discrete time ISS-Lyapunov function as specified by (11) (input being the parameter uncertainty $\|\nu\|_{\max }$ ). Similar to the proof of Theorem 2 in [37, we pick a large enough $\sigma$ to ensure the matrix $\Lambda=\left[\begin{array}{cc}a_{1} & -a_{2} \\ -a_{2} & a_{4}\end{array}\right]$ is positive definite. A specific value for $\sigma$ is given in page 9 of [37] that yields exponential convergence of hybrid periodic orbits. This is extended to include parameter uncertainty by utilizing 111 in 92 in the following manner:

$$
\begin{aligned}
V_{P}(\mathcal{P}(\Theta ; \eta, z))-V_{P}(\eta, z) \leq & -\lambda_{\min }(\Lambda)\|(\eta, z)\|^{2} \\
& +\left(2 a_{3}\|\eta\|+2 a_{5}\|z\|+a_{6}\|\nu\|_{\max }\right)\|\nu\|_{\max } .
\end{aligned}
$$

Therefore, to compute the limit on $\|\nu\|_{\max }$, we can divide the minimum eigen value $\lambda_{\min }(\Lambda)$ by 2 and obtain the following:

$$
\begin{aligned}
& V_{P}(\mathcal{P}(\Theta ; \eta, z))-V_{P}(\eta, z) \leq-\frac{\lambda_{\min }(\Lambda)}{2}\|(\eta, z)\|^{2} \\
& \text { for }-\frac{\lambda_{\min }(\Lambda)}{2}\|(\eta, z)\|^{2}+2\left(a_{3}+a_{5}\right)\|(\eta, z)\|\|\nu\|_{\max }+a_{6}\|\nu\|_{\max }^{2}>0 .
\end{aligned}
$$

Therefore exponential upper bound is obtained from the positive root of the quadratic equation in (95):

$$
\varlimsup_{n \rightarrow \infty}\left\|\mathcal{P}^{n}(\Theta ; \eta, z)\right\| \leq \frac{2\left(a_{3}+a_{5}\right)+\sqrt{4\left(a_{3}+a_{5}\right)^{2}+2 \lambda_{\min } a_{6}}}{\lambda_{\min }}\|\nu\|_{\max },
$$

and we expect this limit to be less than $r$ which yields the upper bound, $\delta_{\max }$, on $\|\nu\|_{\max }$ :

$$
\delta_{\max }:=\frac{r \lambda_{\min }}{2\left(a_{3}+a_{5}\right)+\sqrt{4\left(a_{3}+a_{5}\right)^{2}+2 \lambda_{\min } a_{6}}}\|\nu\|_{\max } .
$$

Therefore, we take the minimum value for the upper bound from 82 , 83, , (97) to obtain $\delta=\min \left\{\delta_{r}, \delta_{I}, \delta_{\max }\right\}$, resulting in exponential parameter to state stability of $\mathcal{O}$. 


\section{Simulation Results}

In this section, we will investigate how the uncertainty in parameters affects the stability of the controller applied to the 5-DOF bipedal robot AMBER shown in Fig. 1. The model, $\Theta_{a}$, which has 61 parameters is picked such that the error is $30 \%$ compared to the assumed model $\hat{\Theta}$.

To realize walking on the robot, the actual and desired outputs are chosen as in 45] (specifically, see (6) for determining the actual and the desired outputs). The end result is outputs of the form $y(q)=y_{a}(q)-y_{d}(q)$ which must be driven to zero. Therefore, the objective of the controller (47) with $\mu(\eta) \in K_{\varepsilon}(\eta)$ and $375 \bar{\mu}(\eta) \in \bar{K}_{\varepsilon, \bar{\varepsilon}}(\eta)$ is to drive $y \rightarrow 0$. For the nominal model, $\hat{\Theta}$, a stable walking gait is observed. In other words, a stable hybrid periodic orbit is observed for the assumed given model. Since, the actual model of the robot has an error of $30 \%$, applying the controller yields the dynamics that evolves as shown in (49). The value of $\varepsilon$ chosen was 0.1 , and $\bar{\varepsilon}$ was 10 . Fig. 2 shows the comparison between actual and desired outputs, and Fig. 3 shows the Lyapunov function $V_{\varepsilon}$. It can be observed that $V_{\varepsilon}$ is always within the bound $\approx 0.06$, while $\|\nu\|$ takes large values at the beginning and end of every step (implying that the control effort applied is large during those points).
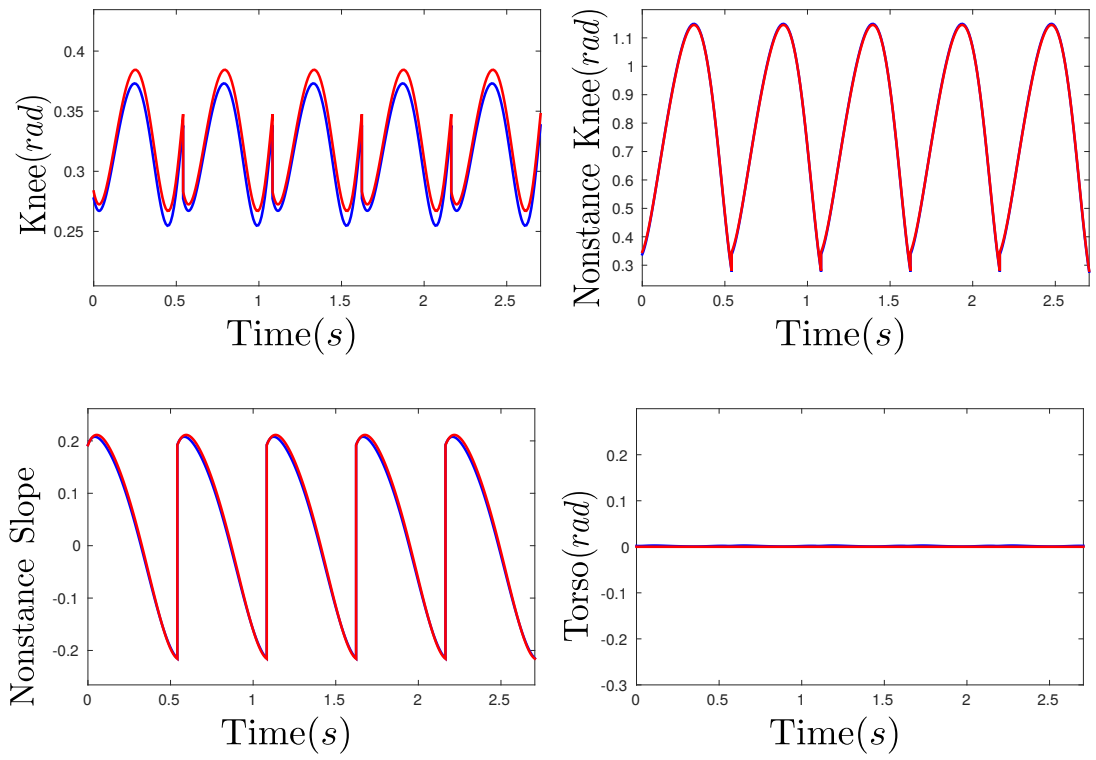

Figure 2: Actual (blue) and desired (red) outputs as a function of time are shown here. Each figure corresponds to an output described to the left of the figures.

Fig. 4 shows the progression of the CLF over two steps with different values of the auxiliary gain $\bar{\varepsilon}$. Value of $\varepsilon$ chosen was 0.05 . Plots are shown for the first two steps as opposed to the steady state behavior shown in Fig. 3. The measure 


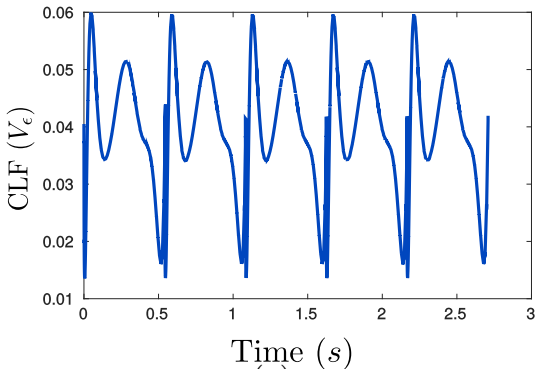

(a)

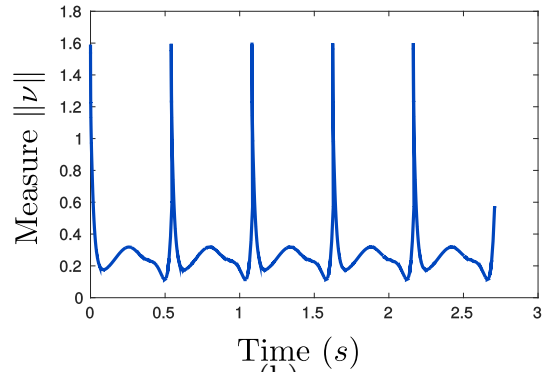

(b)

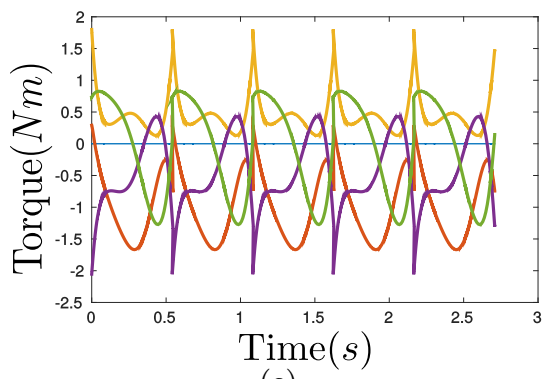

(c)

Figure 3: The RES-CLF (a), the measure (b) and the torque (c) as a function of time. $\dot{V}_{\varepsilon}$ (slope of $V_{\varepsilon}$ ) crosses 0 in every step, but the CLF is still seen to be ultimately bounded. It can also be observed that $\|\nu\|$ increases when the torque inputs are high.

norm $\|\nu\|_{\infty}$ remains consistent (except during impacts) for different values of $\bar{\varepsilon}$, resulting in lower ultimate bounds.
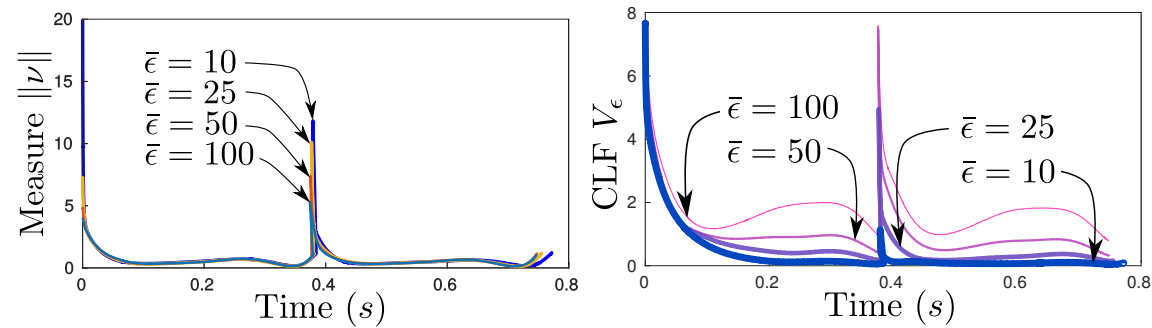

Figure 4: Progression of the measure, RES-CLF from the same initial point for different values of the auxiliary gain $\bar{\varepsilon}$ is shown in the figure. It appears that the ultimate bound decreases with the auxiliary gain $\bar{\varepsilon}$, thereby nullifying the effect of uncertain dynamics.

\section{Conclusions}

390

The concept of a path dependent measure for evaluating the parameter uncertainty of hybrid systems models of robots was introduced. The main formulation of the paper was parameter to state stability which quantifies the affect 
of parameter uncertainty on the performance of the system. Utilizing this notion, coupled with rapidly exponentially stabilizing control Lyapunov functions (RES-CLFs), we are able to establish the main results of the paper: conditions on parameter to state stability of both continuous and hybrid systems. Concretely, these results were applied to the case when the there is a stable periodic orbit in the zero dynamics, therein implying a stable periodic orbit in the full order dynamics even in the case of parameter uncertainty. This was then ver-

400 ified by realizing a stable walking gait on AMBER having a parameter error of $30 \%$. It is important to observe that while the parameter uncertainty measure yields the difference between the actual and the predicted torque applied on the robot, the impact measure yields the difference between the actual and predicted impulsive ground reaction forces acting upon the robot. Therefore, this can be used as an effective tool to not only design the robot model more effectively, but also to design the nominal trajectories for the robots that takes the least path of the measure.

Acknowledgements. This work is supported by the National Science Foundation through grant NRI 1526519.

\section{References}

[1] S. Kolathaya, A. D. Ames, Parameter sensitivity and boundedness of robotic hybrid periodic orbits, IFAC-PapersOnLine 48 (27) (2015) 377382.

[2] P. K. Khosla, Categorization of parameters in the dynamic robot model, Robotics and Automation, IEEE Transactions on 5 (3) (1989) 261-268.

[3] A. Goswami, A. Quaid, M. Peshkin, Complete parameter identification of a robot from partial pose information, in: Robotics and Automation, 1993. Proceedings., 1993 IEEE International Conference on, 1993, pp. 168-173 vol.1. doi:10.1109/ROBOT.1993.291978.

[4] H. Park, K. Sreenath, J. Hurst, J. W. Grizzle, Identification of a bipedal robot with a compliant drivetrain: Parameter estimation for control design, IEEE Control Systems Magazine 31 (2) (2011) 63-88.

[5] S. Gutman, G. Leitmann, Stabilizing control for linear systems with bounded parameter and input uncertainty, in: Optimization Techniques Modeling and Optimization in the Service of Man Part 2, Springer, 1975, pp. 729-755.

[6] D. M. Dawson, Z. Qu, On the global uniform ultimate boundedness of a dcal-like robot controller, Robotics and Automation, IEEE Transactions on 8 (3) (1992) 409-413.

430

[7] H. Berghuis, H. Nijmeijer, Robust control of robots via linear estimated state feedback, Automatic Control, IEEE Transactions on 39 (10) (1994) 2159-2162. doi:10.1109/9.328807. 
[8] Z. Qu, J. Dorsey, Robust tracking control of robots by a linear feedback law, Automatic Control, IEEE Transactions on 36 (9) (1991) 1081-1084. doi:10.1109/9.83543

[9] J.-J. E. Slotine, W. Li, On the adaptive control of robot manipulators, The International Journal of Robotics Research 6 (3) (1987) 49-59.

[10] R. Ortega, M. W. Spong, Adaptive motion control of rigid robots: A tutorial, Automatica 25 (6) (1989) 877-888.

[11] M. Takegaki, S. Arimoto, A new feedback method for dynamic control of manipulators, Journal of Dynamic Systems, Measurement, and Control 103 (2) (1981) 119-125.

[12] S. Arimoto, Stability and robustness of pid feedback control for robot manipulators of sensory capability, International Journal of Robotics Research (1984) 783-799.

[13] P. Tomei, Adaptive pd controller for robot manipulators, Robotics and Automation, IEEE Transactions on 7 (4) (1991) 565-570. doi:10.1109/ 70.86088 .

[14] R. Ortega, A. Loria, R. Kelly, A semiglobally stable output feedback pi2d regulator for robot manipulators, Automatic Control, IEEE Transactions on 40 (8) (1995) 1432-1436. doi:10.1109/9.402235.

[15] R. Kelly, Global positioning of robot manipulators via pd control plus a class of nonlinear integral actions, Automatic Control, IEEE Transactions on 43 (7) (1998) 934-938. doi:10.1109/9.701091.

[16] D. Angeli, Input-to-state stability of pd-controlled robotic systems, Automatica 35 (7) (1999) 1285-1290.

[17] Y. Choi, W. K. Chung, I. H. Suh, Performance and h infin; optimality of pid trajectory tracking controller for lagrangian systems, Robotics and Automation, IEEE Transactions on 17 (6) (2001) 857-869. doi:10.1109/ 70.976011.

[18] R. Beerens, Adaptive control on bipedal humanoid robots.

[19] Z. Li, S. S. Ge, Adaptive robust controls of biped robots, IET Control Theory \& Applications 7 (2) (2013) 161-175.

[20] Q. Nguyen, K. Sreenath, L 1 adaptive control for bipedal robots with control lyapunov function based quadratic programs, in: 2015 American Control Conference (ACC), IEEE, 2015, pp. 862-867.

[21] J. Luo, C. Cao, N. Hovakimyan, L 1 adaptive controller for a class of systems with unknown nonlinearities, in: Proceedings of the 2010 American Control Conference, IEEE, 2010, pp. 1659-1664. 
[22] A. D. Ames, K. Galloway, J. Grizzle, Control lyapunov functions and hybrid zero dynamics, in: Decision and Control (CDC), 2012 IEEE 51st Annual Conference on, IEEE, 2012, pp. 6837-6842.

[23] S. Kolathaya, A. D. Ames, Exponential convergence of a unified clf controller for robotic systems under parameter uncertainty, in: American Control Conference (ACC), 2014, IEEE, 2014, pp. 3710-3715.

[24] E. D. Sontag, Input to state stability: Basic concepts and results, in: Nonlinear and optimal control theory, Springer, 2008, pp. 163-220.

[25] Q. Nguyen, K. Sreenath, Optimal robust control for bipedal robots through control lyapunov function based quadratic programs, in: Robotics: Science and Systems (RSS), Rome, Italy, 2015.

[26] E. D. Sontag, Smooth stabilization implies coprime factorization, Automatic Control, IEEE Transactions on 34 (4) (1989) 435-443.

[27] C. Cai, A. R. Teel, Results on input-to-state stability for hybrid systems, in: Decision and Control, 2005 and 2005 European Control Conference. CDC-ECC'05. 44th IEEE Conference on, IEEE, 2005, pp. 5403-5408.

[28] J. P. Hespanha, D. Liberzon, A. R. Teel, Lyapunov conditions for input-tostate stability of impulsive systems, Automatica 44 (11) (2008) 2735-2744.

[29] J. P. Hespanha, D. Liberzon, A. R. Teel, On input-to-state stability of impulsive systems, in: Decision and Control, 2005 and 2005 European Control Conference. CDC-ECC'05. 44th IEEE Conference on, IEEE, 2005, pp. 3992-3997.

[30] C. Cai, A. R. Teel, Characterizations of input-to-state stability for hybrid systems, Systems \& Control Letters 58 (1) (2009) 47-53.

[31] A. D. Ames, First steps toward automatically generating bipedal robotic walking from human data, in: Robotic Motion and Control 2011, Vol. 422 of LNICS, Springer, 2012, pp. 89-116.

[32] A. D. Ames, S. Sastry, A homology theory for hybrid systems: Hybrid homology, in: Hybrid Systems: Computation and Control, Springer, 2005, pp. 86-102.

[33] A. D. Ames, Characterizing knee-bounce in bipedal robotic walking: A zeno behavior approach, in: Proceedings of the 14th International Conference on Hybrid Systems: Computation and Control, HSCC '11, ACM, New York, NY, USA, 2011, pp. 163-172. doi:10.1145/1967701.1967726.

URL http://doi .acm .org/10.1145/1967701.1967726

[34] E. R. Westervelt, J. W. Grizzle, C. Chevallereau, J. H. Choi, B. Morris, Feedback Control of Dynamic Bipedal Robot Locomotion, CRC Press, Boca Raton, 2007. 
[35] S. Sastry, Nonlinear systems: analysis, stability, and control, Vol. 10, Springer Science \& Business Media, 2013.

[36] R. A. Freeman, P. V. Kokotovic, Robust nonlinear control design: statespace and Lyapunov techniques, Springer, 2008.

[37] A. Ames, K. Galloway, K. Sreenath, J. Grizzle, Rapidly exponentially stabilizing control lyapunov functions and hybrid zero dynamics, Automatic

n Control, IEEE Transactions on 59 (4) (2014) 876-891. doi:10.1109/TAC. 2014.2299335

[38] M. W. Spong, S. Hutchinson, M. Vidyasagar, Robot modeling and control, John Wiley \& Sons Hoboken^eNJ NJ, 2006.

[39] D. G. Cacuci, M. Ionescu-Bujor, I. M. Navon, Sensitivity and uncertainty analysis, volume II: applications to large-scale systems, Vol. 2, CRC press, 2005 .

[40] J. Mulero-Martinez, Uniform bounds of the coriolis/centripetal matrix of serial robot manipulators, Robotics, IEEE Transactions on 23 (5) (2007) 1083-1089. doi:10.1109/TRO.2007.903820.

[41] P. J. From, I. Schjølberg, J. T. Gravdahl, K. Y. Pettersen, T. I. Fossen, On the boundedness and skew-symmetric properties of the inertia and coriolis matrices for vehicle-manipulator systems, in: Intelligent Autonomous Vehicles, Vol. 7, 2010, pp. 193-198.

[42] J. Hauser, C. C. Chung, Converse lyapunov functions for exponentially stable periodic orbits, Systems \& Control Letters 23 (1) (1994) 27-34.

[43] J. W. Grizzle, C. Chevallereau, A. D. Ames, R. W. Sinnet, 3d bipedal robotic walking: models, feedback control, and open problems, IFAC Proceedings Volumes 43 (14) (2010) 505-532.

[44] A. Hereid, E. A. Cousineau, C. M. Hubicki, A. D. Ames, 3d dynamic walking with underactuated humanoid robots: A direct collocation framework for optimizing hybrid zero dynamics, in: 2016 IEEE International Conference on Robotics and Automation (ICRA), 2016, pp. 1447-1454. doi:10.1109/ICRA.2016.7487279.

[45] S. N. Yadukumar, M. Pasupuleti, A. D. Ames, From formal methods to algorithmic implementation of human inspired control on bipedal robots, in: Algorithmic Foundations of Robotics X, Springer, 2013, pp. 511-526. 